\title{
Abstracts of the 25th European Workshop on Neonatology
}

\author{
Westerburg Castle, Germany, \\ May 28th - 31st, 2017
}



IOS Press

\section{THE PREVALENCE OF CONGENITAL MALFORMATIONS IN BABIES BORN IN A UNIVERSITY HOSPITAL (INSTANBUL) BETWEEN 2005 - 2014}

\author{
M. Ozdil ${ }^{1}$, B. Tuysuz ${ }^{1}$, Y. Perk ${ }^{1}$, R. Madazl ${ }^{1}$, \\ A.F. Aksoy ${ }^{1}, \underline{\text { M. Vural }}{ }^{1}$ \\ ${ }^{1}$ Istanbul University, Cerrahpaşa Faculty of \\ Medicine, Turkey
}

Background: Congenital malformations carry the risk of recurrence in following pregnancies besides the effect on life span and quality. The aim of the study was to define and determine the incidence of congenital malformations, the distribution of the anomalies according to pathogenesis and clinical features and to search the factors affecting the frequency of congenital anomalies in live births, stillbirths and termination of pregnancy cases.

Methods: Pre and postnatal characteristics of cases, and type of malformation in live birth cases were determined. An autopsy was conducted in stillbirths and terminated pregnancies due to malformations, the cases were evaluated by geneticist and perinatal pathologist and double and triple anomalies were detected in autopsy cases not reported to date.

Results: The study included 12053 cases consisting of 11.434 live births, 256 stillbirths, 224 termination of pregnancy cases and 139 cases who died in the first seven days of life. The incidence of malformations in live births was $2.6 \%$. The incidence raised to $4.1 \%$ if stillbirth and termination of pregnancy cases were added. $68 \%$ of all malformations were isolated, the remaining 32\% multiple malformations. The most common single malformation was a neural tube defect. Chromosomal anomalies were the commonest among multiple anomalies, Down syndrome being the most frequent, followed by single gene diseases.

Conclusions: The exact clinical and genetic diagnosis has significance both in decision of prenatal termination of pregnancy, treatment and prognosis of live cases and in preventive medicine in relation to prenatal diagnosis of following pregnancies.

\section{TRANSPOSITION OF THE GREAT ARTERIES IN NEONATES: INCIDENCE AND OUTCOME}

\author{
A. Smildzere ${ }^{1}$,E. Ligere ${ }^{2}$, D. Kviļuna ${ }^{2}$
}

${ }^{1}$ Clinic of Neonatology University Children's

Hospital, Riga, Latvia

${ }^{2}$ Clinic of Pediatric Cardiology and Cardiac

Surgery, University Children's Hospital, Riga,

Latvia
Background: Transposition of great arteries (TGA) is one of the most common and severe congenital heart diseases (CHD). If managed with early surgical intervention, TGA is a prevalent congenital heart defect with a high survival rate and a good long-term outcome. Our objective was to describe the incidence of TGA, patient characteristics and outcomes and to identify possible predictors of early morbidity and mortality.

Methods: Retrospective analysis through review of clinical and surgical charts of patients with TGA admitted to the Clinic of Neonatology during 2007-2015.

Results: We enrolled 22 neonates, 7 girls and 15 boys in the study with median gestational age of 40 weeks (Range $32<41$ weeks) and body weight (median) of $3.7 \mathrm{~kg}$ (Range $1.63<4.25 \mathrm{~kg}$ ). Prenatal diagnosis in survivors was established in $23.7 \%$ vs. $11 \%$ in nonsurvivors $(p=0.0008)$. Rashkind procedure was performed in 15 patients (68.2\%), 18 hours (median) after the birth (range 6 hours $<22$ days). Balloon atrial septostomy was performed 14 hours after the birth in survivors vs. 62 hours in nonsurvivors $(p<0,05) .16$ neonates had undergone arterial switch operation. In this group we recorded the duration of cardiopulmonary bypass, aortic cross-clamp time, duration of postoperative mechanical ventilation, inotropic treatment, and length of hospital stay. There was no difference in body weight, gestational age, start of treatment in survivors and nonsurvivors. Survival rate was $59 \%$ (13 of 22 patients).

Conclusions: Prenatal diagnosis and early transport with appropriate pharmacological management to the tertiary care center is a major determinant of survival in newborns with TGA.

\section{VARIATIONS OF CEREBRAL BLOOD FLOW IN NEONATES WITH TRANSPOSITION OF GREAT ARTERIES}

M. Cucerea ${ }^{1,2}$, L. Suciu ${ }^{1}$, E. Moldovan ${ }^{3}$, R. Marian ${ }^{1}$, M. Simon ${ }^{1,2}$

${ }^{1}$ University of Medicine and Pharmacy Tîrgu

Mureş, Romania

${ }^{2}$ County Emergency Hospital Tîrgu Mureş,

Regional Neonatal Intensive Care Department,

Romania

${ }^{3}$ Emergency Institute of Cardiovascular Diseases and Transplant Tîrgu Mureş, Romania

Background: The preoperative treatment of transposition of great arteries (TGA) with prostaglandin E1 (PGE1) alone or associated with balloon atrial septostomy (BAS) influences cerebral hemodynamics. The aim of our study was to assess cerebral hemodynamics with Doppler ultrasonography and find correlations between the supposed changes of cerebral hemodynamic 
parameters and PGE1 treatment and BAS in newborns with TGA admitted in our unit, between January 1, 2015 and December 31, 2016.

Methods: We studied cerebral blood flow velocities and resistivity index measured in anterior cerebral artery by colored and pulsed Doppler ultrasonography in newborns $(n=23)$ with TGA before and in the first 6 hours after PGE1 infusion has been started, and before and in the first 6 hours after BAS has been performed.

Results: Out of 23 newborns with gestational age $38.7 \pm 1.5($ mean \pm SD) weeks, $47.8 \%$ were diagnosed prenatally. BAS was performed in 17 of the 23 patients. Systolic velocities (Sv) $(t=3.64, p=0.00074)$ and diastolic velocities (Dv) $(t=-8.64, p<<0.01)$ showed significant decreases after starting PGE1 infusion and resistivity index (RI) has significantly increased $(t=-8.64$, $p<<0.01)$. When given only PGE1 infusion Sv values decreased significantly more and in the case of newborns with BAS, Sv values tend to rise or show a lower decrease compared with those who received only PGE1 infusion $(p=0.0004)$. The same results are also found for Dv after BAS. In newborns with BAS, RI values tend to decline more but insignificant compared with those who received only PGE1 infusion.

Conclusions: Successful BAS improve cerebral hemodynamics in newborns with TGA.

\section{INTRAVENOUS ADMINISTRATION OF HUMAN UMBILICAL CORD-DERIVED MESENCHYMAL STEM CELLS DECREASES PRESSOR USE AND FLUID REQUIREMENTS IN PRETERM BABOONS OF BORDERLINE VIABILITY}

M.A. Möbius 1,2,3,4 , S.B. Mustafa², D.C. McCurnin², C.L. Blanco ${ }^{2}$, D. Freund ${ }^{4}$, B. Thébaud ${ }^{3,5}$, M. Rüdiger ${ }^{2,4}$, S.R. Seidner ${ }^{1}$

\section{${ }^{1}$ Neonatology, Universitätsklinikum "Carl Gustav \\ Carus" Dresden, Saxony, Germany \\ ${ }^{2}$ Pediatrics, UTHSCSA, San Antonio, Texas, USA \\ ${ }^{3}$ Sinclair Centre for Regenerative Medicine, OHRI, \\ Ottawa, Ontario, Canada \\ ${ }^{4}$ DFG Cluster of Excellence for Regenerative \\ Therapies Dresden - CRTD, Technische Universität \\ Dresden, Saxony, Germany \\ ${ }^{5}$ Pediatrics, Children's Hospital of Eastern Ontario, Ottawa, Ontario, Canada}

Purpose of Study: To evaluate therapeutic effects of mesenchymal stem cells (MSC) derived from human umbilical cords when given intravenously to preterm baboons electively delivered at borderline viability.

Methods: Premature baboons were delivered by C-section at $125 \pm 2 \mathrm{~d}$ gestational age (full term $=185$ days). Immediately after delivery, animals were intubated and given surfactant. Umbilical arterial catheter and peripherally inserted central catheters were placed. The animals were mechanically ventilated for 14 days with the ventilators adjusted to maintain $\mathrm{paCO}_{2}$ targets of 45-55 $\mathrm{mmHg}(6-7.33 \mathrm{kPa})$ and $\mathrm{paO}_{2}$ targets of $50-70 \mathrm{mmHg}$ (6.6-9.3 kPa). At $2 \mathrm{~h}$ of life, $1.5 \mathrm{ml}$ of MSC were administered over $15 \mathrm{~min}$ in a dose of $10^{7}$ cells $/ \mathrm{kg}$ birthweight to treated animals versus vehicle to controls. Results are expressed as group means \pm SD.

Results: 4 untreated controls $(2 \mathrm{M}, 2 \mathrm{~F})$ with mean birthweights of $357 \pm 17 \mathrm{~g}$ and $5 \mathrm{MSC}$-treated preterm baboons $(2 \mathrm{M}, 3 \mathrm{~F})$ born at $381 \pm 26 \mathrm{~g}$ survived to $14 \mathrm{~d}$ in stable condition. Despite receiving increased fluid intake from $0-72$ hours of life $(245 \pm 25 \mathrm{ml} / \mathrm{kg} / \mathrm{d}$ in controls vs. $211 \pm 9 \mathrm{ml} / \mathrm{kg} / \mathrm{d}$ in MSC-treated), 3 out of 4 controls required dopamine vs. 0 out of 5 MSC-treated and still had lower mean BPs at $72 \mathrm{~h}(35.0 \pm 7.2 \mathrm{mmHg}$ vs. $40.0 \pm 4.7$ $\mathrm{mmHg}$ ). Average urine output from $0-72 \mathrm{~h}$ was similar in controls vs. MSC-treated $(5.4 \pm 0.4 \mathrm{ml} / \mathrm{kg} / \mathrm{h}$ vs. $4.6 \pm 0.8$ $\mathrm{ml} / \mathrm{kg} / \mathrm{h}$ ), but controls increased $11 \%$ from birthweight vs. $5 \%$ for MSC-treated. Serum albumin dropped to $<65 \%$ of birth values over the first $72 \mathrm{~h}$ in 3 out of 4 controls vs. only $1 / 5$ MSC-treated.

Conclusions: Treatment of preterm baboons of borderline viability with i.v. MSC appears to decrease capillary leak and increase intravascular volume leading to improved hemodynamic stability and less need for supplemental pressors during the critical first $72 \mathrm{~h}$ of life.

\section{MATERNAL SMOKING IS ASSOCIATED WITH CHANGES IN TIDAL BREATHING PATTERN IN HEALTHY NEWBORNS}

\author{
Z. Gyurkovits ${ }^{1}$, B. Radics ${ }^{2}$, Z. Gingl ${ }^{3}$, G. Makan ${ }^{3}$,
} H. Orvos ${ }^{1}$, G. Németh ${ }^{1}, Z$. Hantos ${ }^{2}$

${ }^{1}$ Department of Obstetrics and Gynecology,

University of Szeged, Hungary

${ }^{2}$ Department of Pulmonology, University of Szeged,

Hungary

${ }^{3}$ Department of Technical Informatics, University of Szeged, Hungary

Background: Several prenatal risk factors can affect the lung function in infants. Our study focused on the influence of maternal smoking on respiratory mechanics in spontaneously breathing newborns.

Methods: Healthy term newborns (exposed to maternal smoke [Group S], $n=13$; controls [Group C], $n=16$ ) were measured with the forced oscillation technique during natural sleep within 24 hours after birth. Within-breath changes in the mechanical impedance of the respiratory system $\left(Z_{\mathrm{rs}}\right)$ were estimated at $16 \mathrm{~Hz}$ and spirogram was registered with a pneumotachograph. The average of minimum 5 breaths from steady-state periods was 
calculated for statistical analysis. Mann-Whitney Test and Analysis of Covariance were employed to examine the effects of maternal smoking on the tidal breathing parameters and the components of $\mathrm{Z}_{\mathrm{rs}}$.

Results: There was no difference in anthropometric data or in respiratory rate (data: median [25th-75th percentile] 63 [52-74] vs 66 [58-73] $\mathrm{min}^{-1}$ ) between Groups $\mathrm{C}$ and $\mathrm{S}$. Tidal volume and respiratory rate were inversely proportional in Group $\mathrm{C}\left(r^{2}=0.67\right)$ but were uncorrelated in Group S $\left(r^{2}=0.01, \mathrm{p}<0.05\right)$.

The ratio of time to peak expiratory flow and total expiratory time $\left(\mathrm{T}_{\mathrm{PTEF}}: \mathrm{T}_{\mathrm{E}}\right)$ was significantly $(\mathrm{p}<0.05)$ lower in Group S (0.51 [0.43-0.59] vs 0.44 [0.38-0.49]). No difference was found in any examined parameters of $\mathrm{Z}_{\mathrm{rs}}$ between Groups C and S.

Conclusions: Maternal smoking affects tidal breathing in healthy newborns via altered central neural control of breathing, while these changes are not associated with any impairment in lung mechanical properties. Nicotine, as a known stimulant of cholinergic transmission might be responsible for these changes.

\section{METABOLOMIC APPROACH TO INHERITED ERRORS OF METABOLISM IN NEONATAL AGE}

N. Jekova ${ }^{1}$, M. Ivanova ${ }^{2}$

${ }^{1}$ Department of Neonatology, University hospital of Obstetrics and Gynecology, Sofia

${ }^{2}$ National Genetic Laboratory, University hospital of Obstetrics and Gynecology, Sofia

Background: Organic acidurias are a clinically important heterogeneous group of rare inherited disorders. The diagnosis requires an application of many highly specialized methods and a comprehensive approach because of their unspecific clinical manifestation. Here we present the metabolomic approach to the diagnosis of inherited errors of metabolism manifested in the neonatal period.

Methods: The metabolomic approach includes the following steps of investigation: urine qualitative target analysis of certain metabolites, mass spectrometry (MS) of blood spot aminoacid and acyl-carnitine profiles, gas chromatography (GC) / MS of urine organic acid profile and DNA analysis for confirmation of diagnosis. The main analytical techniques in our approach were the tandem MS / MS and GC / MS.

Results: More than 1500 high risk neonates were investigated by using a metabolomic study. A total of 126 infants $(8.8 \%)$ were diagnosed and classified into eleven basic organic acid disorders' groups as follows: defects of aromatic amino-acid metabolites $(n=13)$, defects of propionate and methylmalonate metabolism $(\mathrm{n}=8)$, branched chain amino-acid metabolism ( $\mathrm{n}=13)$, mitochondrial fatty acid oxidation $(n=14)$, pyrimidine metabolism $(\mathrm{n}=1), \gamma$-glutamyl cycle defects $(\mathrm{n}=2)$, dibasic aminoacid metabolism $(\mathrm{n}=2)$, lactic aciduria $(\mathrm{n}=$ $38)$, defects of glycolysis and Krebs' cycle $(n=8)$, urea cycle $(\mathrm{n}=21)$, and miscellaneous inherited metabolic disorders $(\mathrm{n}=5)$. The diagnostically informative metabolite profiles were detected for each disease.

Conclusion: Our results show that the application of a metabolomic approach is a useful, rapid and feasible tool for diagnosis of inherited organic acidurias.

\section{TREATMENT FAILURE FOLLOWING LESS INVASIVE SURFACTANT ADMINISTRATION (LISA)}

\section{E. Herting ${ }^{1}$, A. Kribs ${ }^{2}$ and W. Göpel ${ }^{1}$ \\ Departments of Pediatrics, Universities of Lübeck ${ }^{1}$ and Cologne ${ }^{2}$, Germany}

Background: LISA is a procedure that accounts for more than $50 \%$ of surfactant treatments in Germany by now. We analyzed the success rate (avoidance of mechanical ventilation and prevention of BPD) in different gestational ages.

Methods: The GNN is a prospective cohort $(n>15.000)$ study of preterm infants $<1500 \mathrm{~g}$.

Results: 3108 infants that underwent LISA were analyzed (see below).

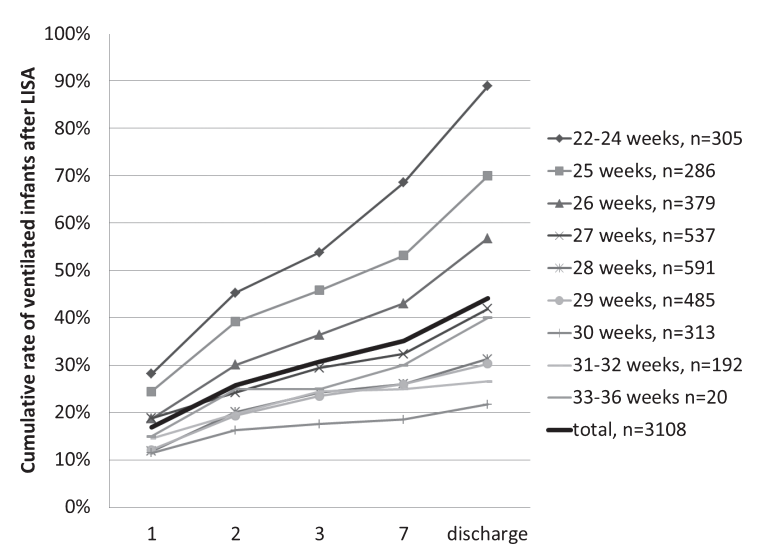

Conclusions: Treatment response following LISA depends on gestational age both in terms of prevention of mechanical ventilation and BPD. 


\section{AN OPEN LABEL, DOSE-ESCALATION STUDY OF LUCINACTANT FOR INHALATION DELIVERED VIA NASAL CONTINUOUS POSITIVE AIRWAY PRESSURE (NCPAP) FOR TREATING RESPIRATORY DISTRESS SYNDROME (RDS) IN PRETERM NEONATES}

\author{
J. Mazela ${ }^{1}$, N.N. Finer ${ }^{2}$, S.G. Simonson ${ }^{3}$, \\ P.M. Shore ${ }^{3}$, P. Simmons ${ }^{3}$, R. Segal ${ }^{3}$ \\ ${ }^{1}$ Poznan University of Medical Sciences in Poznan, \\ Department of Neonatology and Infectious \\ Diseases, Poznan, Poland \\ ${ }^{2}$ Sharp Mary Birch Hospital for Women and \\ Newborns, Neonatal Research Institute, San Diego, \\ CA, USA
}

${ }^{3}$ Windtree Therapeutics, Inc, Warrington, PA, USA

Introduction: nCPAP supports respiration but limits early surfactant replacement therapy (SRT) to preterm infants. Aerosolized surfactant via nCPAP could avoid endotracheal intubation (ET) for SRT delivery but has had limited success. Lucinactant for Inhalation is a novel drug-device therapy that delivers an aerosolized SRT using proprietary capillary aerosol-generating technology.

Methods: We conducted a phase 2 dose-escalation study to assess the safety/tolerability of Lucinactant for Inhalation in preterm infants of 29-34 completed weeks post menstrual age (PMA) with RDS. Adverse events (AEs), including common complication of prematurity, CPAP failure, oxygen requirements and physiological parameters were assessed.

Results: 80 neonates were sequentially enrolled into 5 dosing groups $(25,50,75,100$ and $150 \mathrm{mg}$ total phospholipids [TPL] $/ \mathrm{kg} ; 8$ treated and 8 nCPAP-only control subjects/group). The 3 most common AEs were neonatal jaundice, constipation, and apnea and were comparable between groups. Air leak was the most common complication of prematurity in both treatment groups. $\mathrm{FiO}_{2}$ decreased from baseline more rapidly with Lucinactant for Inhalation $(30 \mathrm{~min})$ than in controls (3 hours). The dose response for preventing nCPAP failure appeared to be relatively flat above $50 \mathrm{mg}$ TPL $/ \mathrm{kg}$. nCPAP failure requiring rescue therapy occurred in $30 \%$ of Lucinactant for Inhalation-treated infants in the 3 highest dose groups (7/23) versus 53\% (21/40) in all controls.

Conclusions: Lucinactant for Inhalation was welltolerated via nCPAP in preterm infants 29-34 weeks PMA with RDS and may provide an alternative to surfactant administration via an ET tube.

Supported in part through NIH/NHLBI SBIR 4R44HL107000-02

\section{IN VITRO MEASUREMENT OF PRESSURE AND FLOW CHARACTERISTICS OF DIFFERENT NASAL CONTINUOUS POSITIVE AIRWAY PRESSURE DEVICES FOR NEONATES}

$\underline{T}^{\text {Juren }}{ }^{1}$, F. Lizal ${ }^{2}$, M. Pohorilsky²

${ }^{1}$ Department of Neonatology, University Hospital
Brno, Czech Republic
${ }^{2}$ Faculty of Mechanical Engineering, Brno
University of Technology, Czech Republic

Background: Our aim was to compare devices for nasal continuous positive airway pressure (NCPAP) application in neonates by measuring the pressure of the device and flow delivered to the patient.

Methods: The following NCPAP delivery devices were tested: [Babyflow-Draeger (sizes XS, S, M, L, XL), Vygon single tube $2.5 \mathrm{~mm}$, Vygon double tube $1.5 \mathrm{~mm}$, RAM cannula (for newborns under $1.0 \mathrm{~kg}$ ), Argyle pharyngeal cannula]. The generated flow was set to 8 liter/min (10 liter/min for L and XL), positive endexpiratory pressure was set to 5 mbar ( 6 mbar for $\mathrm{L}$ and $\mathrm{XL})$. A flow to the patient was calculated as a difference of flow at the inspiratory and expiratory arm of the Babylog-Draeger ventilator circuit; the pressure was measured at the proximal end of each cannula by a calibrated manometer.

Results: The pressure (in mbar) was: Vygon single: 2.29; Vygon double: 3.15; RAM cannula: 1.43; Argyle pharyngeal: 4,71; Babyflow-Draeger: (XS) 2.32; (S) 1.86; (M) 1.05; (L) 0.86; (XL) 0.65. The flow (liter/min) to patient was: Vygon single: 3.25; Vygon double: 2.81; RAM cannula: 4.10; Argyle pharyngeal: 2.93; BabyflowDraeger: (XS) 6.06; (S) 7.87; (M) 7.8.

Conclusions: High pressure at the proximal end of a device indicates high resistance, which decreases the flow to patient. Generally, devices with short tubes and greater diameters imply lower resistance.

\section{DIAGNOSIS OF BRONCHOPULMONARY DYSPLASIA USING NOVEL BIOMARKERS AND MAGNETIC RESONANCE IMAGING ANALYSIS - FINDINGS FROM THE AIRR-STUDY}

\author{
K. Förster ${ }^{1,6}$, S. Sass ${ }^{2}$, H. Ehrhardt ${ }^{3}$, \\ B. Ertl-Wagner ${ }^{4}$, D. S. Mous ${ }^{5}$, R.J. Rottier ${ }^{5}$, P. Oak ${ }^{6}$, \\ A. Pomschar ${ }^{4}$, L. Nährlich ${ }^{3}$, A. Schulze ${ }^{1}$, \\ A.W. Flemmer ${ }^{1}$, J. Gronbach ${ }^{3}$, C. Hübener ${ }^{7}$, \\ O. Eickelberg ${ }^{6}$, O. Dietrich ${ }^{4}$, F.J. Theis ${ }^{2,8}$, \\ A. Hilgendorff,
}


${ }^{1}$ Dept. of Neonatology, Perinatal Center Grosshadern, Dr. von Hauner Children's Hospital, Ludwig-Maximilian-University, Munich, Germany ${ }^{2}$ Institute of Computational Biology, Helmholtz Center, Munich, Germany ${ }^{3}$ Department of General Pediatrics and Neonatology, University Hospital of Giessen and Marburg, Giessen, Germany, Member of the German Lung Research Center (DZL) ${ }^{4}$ Institute for Clinical Radiology, LudwigMaximilian-University Hospital, Munich, Germany, Member of the German Lung Research Center (DZL) ${ }^{5}$ Department of Pediatric Surgery, Erasmus Medical Center-Sophia Children's Hospital, Rotterdam, The Netherlands

${ }^{6}$ Comprehensive Pneumology Center, Helmholtz Center, Munich, Germany, Member of the German Lung Research Center (DZL) ${ }^{7}$ Clinic for Gynecology and Obstetrics, Perinatal Center Grosshadern, Ludwig-Maximilian-

University, Munich, Germany

${ }^{8}$ Department of Mathematics, Technical University of Munich, Germany

Rationale: Neonatal chronic lung disease, i.e. bronchopulmonary dysplasia (BPD) determines long-term pulmonary and neurologic development. Early markers are urgently needed for timely diagnosis and personalized treatment.

Objectives: The prospective study determined structural and functional changes in the preterm lung at the time of diagnosis and identified early disease markers by proteome screening in plasma in the first week of life.

Method: 40 infants $(27.7 \pm 2.09$ wks, $984 \pm 332 \mathrm{~g})$ were included for advanced MRI measurements (3-Tesla) and complemented by infant lung function testing (ILFT) in spontaneously breathing infants. Plasma samples were processed for proteomic screening by SOMAscan ${ }^{\mathrm{TM}}$. Key findings were confirmed in an independent study cohort. Statistical analysis used penalized and Poisson regression analysis; for protein analysis confounder effects were subtracted by lasso regression.

Results: Statistical analysis confirmed a high correlation of MRI and lung function variables and identified a pattern characterizing changes in the lungs of preterm infants by T2- and T1-weighed image analysis and lung volume measurements as well as ILFT. Functional enrichment analysis in proteome screening results showed overrepresentation of the GO categories 'immune function', 'extracellular matrix', 'cellular proliferation/ migration', 'organ development' and 'angiogenesis' in infants with BPD in the first week of life. A panel of three proteins was identified out of these with high sensitivity and specificity for BPD.
Conclusions: We identified a structural pattern characterizing BPD by advanced MRI confirmed by ILFT. The identified development in the first week of life enabled personalized treatment strategies.

\section{TWO YEAR OUTCOME IN FORMER VERY PRETERM NEONATES TREATED WITH LATE SURFACTANT FOR PROLONGED MECHANICAL VENTILATION}

J.M. Hascoët $^{1}$, J.C. Picaud ${ }^{2}$, I. Ligi ${ }^{3}$, T. Blanc ${ }^{4}$, M.F. Pinturier ${ }^{5}$, V. Zupan ${ }^{6}$, F. Moreau ${ }^{7}$, I. Guilhoto ${ }^{8}$, M. Rouabah ${ }^{1}$, C. Alexandre ${ }^{9}$, E. Saliba ${ }^{10}$, L. Storme ${ }^{11}$, J. Patkai ${ }^{12}$, M. Pomedio ${ }^{13}$, I. Hamon ${ }^{1}$

${ }^{1}$ DevAH, Maternite Regionale CHRU, Nancy

${ }^{2}$ CHU Lyon Croix Rousse, France

${ }^{3}$ AP-HM Marseille, France

${ }^{4}$ CHU Rouen, France

${ }^{5} \mathrm{CH}$ Montreuil, France

${ }^{6}$ AP-HP Clamart, France

${ }^{7} \mathrm{CHU}$ Amiens, France

${ }^{8} \mathrm{CH}$ Arras, France

${ }^{9} \mathrm{CHU}$ Caen, France

${ }^{10} \mathrm{CHU}$ Tours, France

${ }^{11}$ CHU Lille, France

${ }^{12}$ AP-HP Paris Port Royal, France

${ }^{13} \mathrm{CHU}$ Reims, France

Background: In a multicenter, randomized, controlled trial, a curative administration of $200 \mathrm{mg} / \mathrm{kg}$ Poractant alfa (CURO) vs Air, in infants with prolonged mechanical ventilation, did not alter the early course of bronchopulmonary dysplasia, but CURO infants had significantly less re-hospitalization for respiratory problems at one year postnatal age (PNA). This study aimed to evaluate the outcome at 2 years PNA of the infants involved in this trial.

Methods: 118 very premature infants were included (mean $=26 \pm 1.4$ weeks of gestational age). All surviving infants were eligible for this follow-up study. After parental interview, infants underwent complete physical examination by certified trained pediatricians not aware of the randomization.

Results: $90 / 96$ eligible infants, CURO $(n=45)$ vs Air $(n=45)$, were evaluated at a similar median [IQR] of 25 [24-27] months. At 2 years PNA, weight and weight z-score were significantly better in CURO infants $(10.9 \pm 1.7$ vs. $10.2 \pm 1.8 \mathrm{Kg} ; p=0.034 ;-0.51 \pm 1.10$ vs. $-0.96 \pm 1.19 ; p=0.025$, respectively (mean $\pm \mathrm{SD})$ ) when it was not different at birth and at 1 year PNA. No difference was observed for height, head circumference or respiratory morbidity. However, infants hospitalized within their first year were more prone to be re-hospitalized during their 
second year $(\mathrm{OR}=4.182[1.478-11.834] p=0.007)$. Neurodevelopmental scores were similar between groups; $93 \%$ of the infants walked at $17.4 \pm 2.8$ vs. $18.4 \pm 2.8$ months PNA, $p=0.110$.

Conclusion: At two years of age, infants treated with late curative surfactant had better growth than controls. There was no significant difference for respiratory morbidity but infants hospitalized within the first year were more prone to re-hospitalization during their second year.

\section{EPIDEMIOLOGY OF BLOOD-STREAM INFECTION IN PORTUGUESE NEONATAL INTENSIVE CARE UNITS}

Principal investigators - Maria Teresa Neto, Alexandra Almeida

Co-investigators: Almerinda Pereira, Ana Lúcia Maia, Cristina Resende, Ermelinda Mendes, Eunice Soares, Olga Voutsen, Margarida Cabral, Luísa Malheiro, Anabela João, Mónica Marçal, Susana Pissarra, Margarida Abrantes, $M^{a}$ José Mendes, Edite Costa, Teresa Cezanne, Teresa Caldeira.

\section{Department of Quality in Health, Directorate General of Health, Lisbon, Portugal}

Objective: To know the epidemiology of bloodstream infection in newborn infants admitted to Portuguese NICUs.

Methods: All patients in the National Registry, admitted from January 2008 to December 2015, with sepsis, meningitis, necrotizing enterocolitis and pneumonia with positive blood or cerebrospinal fluid cultures were enrolled.

Results: There were 40.205 registrations with 2906 positive cultures in 2352 newborn infants accounting for 2593 episodes of infection (10.9 episodes of infection/1000 admission days). The median gestational age and birth weight were 30 weeks (22-42) and 1300g (416-5270); $55.2 \%$ were male; 2273 episodes were sepsis, 121 sepsis with meningitis, 103 necrotizing enterocolitis with sepsis, 95 sepsis with pneumonia and 1 sepsis with meningitis and pneumonia; 433 (16.7\%) episodes were classified as earlyonset, $1742(67.2 \%)$ as late-onset and 418 as post-neonatal infections. There were 141 deaths caused by infection (lethality 6\%). Five cultures grew two different strains total of strains 2598 . The most frequent isolates were coagulase negative Staphylococcus $(91.2 \%$ resistant to methicillin, $81.4 \%$ resistant to gentamicin); $S$. aureus (25.3\% resistant to methicillin); E.coli (68.9\% resistant to ampicillin); group B Streptococcus; Klebsiella spp. (44.4\% resistant to cefotaxime and $33.3 \%$ gentamicin); $41 \%$ of Enterobacter spp. were resistant to cefotaxime; 1551 episodes of infection (with peripheral and/or central venous catheter (CVC) positive blood culture) occurred in newborn infants with a CVC-rate of infection in patients with CVC 10.4/1000 CVC days.

Conclusions: Concerning type of infection and microorganisms, findings are in the range of expected; bacterial resistance is a cause of concern as well as the rate of infection in newborn infants with CVC.

\section{COLONIZATION / SEPSIS DUE TO CENTRAL CATHETERS IN NEWBORNS IN INTENSIVE CARE UNIT}

\author{
C. Cunha ${ }^{1}$, C. Gomes ${ }^{1}$, A. Paredes ${ }^{1}$, F. Amorim ${ }^{1}$, \\ A. Silva ${ }^{2}$, M. Marques ${ }^{2}$ A. Barroso Pereira ${ }^{2}$ \\ Neonatal Intensive Care Unit-Hospital de Braga, \\ Portugal
}

Introduction: Central catheter (CVC) colonization, and sepsis related to $\mathrm{CVC}$, are complications associated with the introduction and maintenance of CVC in newborns (NBs). In 2012 a procedure was elaborated on the introduction and maintenance of CVC in NBs in Hospital de Braga Neonatal Intensive Care Unit (NICU). Aim of the study was to evaluate the infections associated with CVC in the NICU NBs after the use of the CVC procedure.

Methods: Prospective, descriptive study, with database analysis of all NBs with CVC, each year, from 2013 to 2016, at the NICU of Hospital de Braga. We evaluated gestational age (GA), number of CVCs introduced, and catheters tip microbiology and blood cultures.

Results: A total of $383 \mathrm{CVC}$ were introduced in 263 NBs hospitalized at NICU, GA 23 weeks 1 day to 41 weeks, median GA 30 weeks 0 days. The culture of the CVC tips were negative in 71/92(77.17\%), 50/60 (83.33\%), $74 / 82(90.24 \%)$ and 81/101 (80.19\%) from 2013 to 2016. The colonized tips were Staphylococcus epidermidis 10/21 (47.61\%), Enterococcus faecalis 4/21 (10.04\%), Staphylococcus epidermidis 9/10 (90\%), Bacillus licheniformis $1 / 10(10 \%)$, Staphylococcus epidermidis $4 / 8$ (50\%), Staphylococcus werneri $3 / 8 \quad(37.5 \%)$, Staphylococcus epidermidis $9 / 20(45 \%)$ and Candida albicans $4 / 20$ (20\%) from 2013 to 2016 . Only 1 sepsis related to CVC, Klebsiella pneumoniae ssp, was reported in an infant with umbilical catheter in the year 2013.

Conclusion: This study shows that the CVC in NICU procedure, awareness raising, training and updating of the team, as well as colonization / infection monitoring are fundamental in the prevention of $\mathrm{CVC}$ related sepsis. 


\section{NEONATAL GROUP B}

STREPTOCOCCAL MENINGITIS: RETROSPECTIVE ANALYSIS OF DIAGNOSTIC PARAMETERS AND EARLY OUTCOME

\author{
$\underline{\text { P. Fister }}^{1}$, Z. Rener Primec ${ }^{2}$, D. Paro Panjan ${ }^{1}$
}

${ }^{1}$ Clinical Department of Neonatology, University

Children's Hospital, University Medical Centre

Ljubljana, Ljubljana 1000, Slovenia

${ }^{2}$ Clinical Department of Neurology, University

Children's Hospital, University Medical Centre

Ljubljana, Ljubljana 1000, Slovenia

Background: After compulsory vaccination of preschool and school children against Haemophilus influenzae type $\mathrm{b}$ and Streptococcus pneumoniae, group B Streptococcus (GBS) became the most common cause of bacterial meningitis in newborns and infants. It is known that half of the children after acute neonatal GBS meningitis later develop neurological sequelae. Our aim was to determine the neurological and developmental outcome of children who suffered GBS meningitis in the first three months, and to analyze the significance of clinical, laboratory, neurophysiological and imaging investigations.

Methods: Retrospectively we collected perinatal medical history data, clinical signs, laboratory, neurophysiological and imaging reports and short-term outcome for all infants under three months, treated at the University Children's Hospital Ljubljana in the last six years, due to GBS meningitis.

Results: In 2010 - 2015 we treated 19 newborns and infants up to three months due to GBS meningitis, 11 girls. Five newborns were diagnosed within the first six days with an early-onset of meningitis and 14 after seventh day with late-onset. All newborns with early-onset meningitis were born at term and had good outcome at follow-up. Two-thirds of newborns with late-onset meningitis were premature; upon follow-up $8 / 14$ had neurological impairment, two infants died in the second year.

Conclusions: Estimated incidence of GBS meningitis in neonates and infants up to three months in Slovenia is at least 0.16 per 1000 live births. Disturbance of consciousness, convulsions, hemodynamic instability, neutropenia, and abnormal EEG at the beginning of the disease are associated with neurological impairment.

\section{RETROSPECTIVE AUDIT OF MANAGEMENT OF SUSPECTED NECROTIZING ENTEROCOLITIS IN A TERTIARY LEVEL NEONATAL UNIT}

N. Bussmann ${ }^{1}$, A.C. Glynn ${ }^{1}$, S. Ryan ${ }^{1}$, A. Tarrant ${ }^{1}$, A. El Khuffash ${ }^{1}$, J.D. Corcoran ${ }^{1}$
${ }^{1}$ Dept. of Pediatrics, Rotunda Hospital, Dublin and Royal College of Surgeons, Ireland

Necrotizing enterocolitis is a occasionally devastating illness affecting the preterm infant, the risk of which is inversely related to birth weight and gestational age. The diagnosis is based on clinical and radiological findings. However there is poor inter observer agreement between radiologists regarding radiological features of necrotizing enterocolitis which can lead to over diagnosis of necrotizing enterocolitis with resultant overuse of antibiotics and delay in establishing feeds.

We aim to assess the methods and criteria used to diagnose necrotizing enterocolitis in a retrospective study of VLBW born in 2016 utilizing a scoring system that is being evaluated at University College London Hospital (UCLH) which is a modification of the Bell scoring system.

We will assess the subsequent medical management of these cases utilizing the UCLH scoring system to determine if we are overtreating infants for suspected necrotizing enterocolitis.

\section{NEONATAL MANIFESTATIONS OF IMMUNE-MEDIATED RHEUMATIC DISEASES: A RETROSPECTIVE LONGITUDINAL STUDY IN A TERTIARY HOSPITAL}

$\underline{\text { J. Bernardo }}^{1}$, H. Guimarães ${ }^{1,2}$, M. Guimarães ${ }^{1,3}$, R. Fonseca ${ }^{1,4}$, I. Brito $^{1,4}$

${ }^{I}$ Faculty of Medicine, University of Porto, Porto, Portugal

${ }^{2}$ Pediatrics department, São João Hospital Centre, Porto, Portugal

${ }^{3}$ Gynaecology and Obstetrics department, São João

Hospital Centre, Porto, Portugal

${ }^{4}$ Rheumatology department, São João Hospital

Centre, Porto, Portugal

Background: Autoimmune rheumatic diseases such as systemic lupus erythematosus (SLE), antiphospholipid antibody syndrome (APS) and Sjögren's syndrome (SS) may affect women during pregnancy, increasing morbidity and affecting neonatal outcome. Cardiac, cutaneous, hematological, hepatic and pulmonary manifestations may occur. We aim to study the occurrence of pregnancy complications, neonatal lupus and associated factors.

Methods: A retrospective study was performed including pregnant women with immune-mediated rheumatic diseases seen in a multidisciplinary group for autoimmune diseases during pregnancy between 2010 and 2015. Patients were compared using Mann-Whitney, chisquare and Fisher tests (SPSS 24.0), $\alpha<0.05$.

Results: We included 151 gestations of women with SLE, SS, APS, rheumatoid arthritis, Behçet's disease, 
systemic sclerosis, mixed connective tissue diseases and others. During follow-up, $142(94.0 \%)$ babies were born and $7(4.6 \%)$ abortions and $2(1.3 \%)$ stillbirths occurred. $28(19.7 \%)$ were premature $(<37 \mathrm{w})$, and $14(9.9 \%)$ were born with intra-uterine growth restriction (IUGR). Out of $6(4.2 \%)$ babies with neonatal lupus manifestations, $4(66.7 \%)$ were cardiac, $2(33.3 \%)$ were cutaneous, $1(16.7 \%)$ was hepatic, $2(33.3 \%)$ were hematological and $1(16.7 \%)$ was pulmonary; $1(0.7 \%)$ died in uterus with a complete heart block. Prematurity occurred more frequently among neonates with IUGR (53.8\% vs 46.1\%; $p=0.04)$, and was associated with gestational diabetes (21.4\% vs $2.9 \% ; p=0.018)$. It also occurred more frequently in multiple pregnancies $(75 \%$ vs $16.4 \%$; $p=0.001)$, mothers taking glucocorticoids (28.6\% vs $9.2 \% ; p=0.003)$ and active rheumatic disease at conception (23\% vs $6.8 \% ; p=0.03)$. Neonatal lupus manifestations occurred more frequently in mothers with SS $(23.5 \% \mathrm{vs}$ $2.2 \% ; p=0.003)$, anti-SSa/Ro $(20 \%$ vs $0 \% ; p<0.001)$, and anti-SSb/La (27.7\% vs $1.5 \% ; p<0.001)$.

Conclusion: Our study proved a link between immunemediated rheumatic diseases and specific pregnancy outcomes such as prematurity, IUGR and neonatal lupus.

\section{FIRST YEAR OF IMPLEMENTATION NATIONAL REGISTRY OF PERINATAL ASPHYXIA, HYPOXIC-ISCHEMIC ENCEPHALOPATHY (HIE) AND WHOLE-BODY HYPOTHERMIA AT HIE IN SLOVAKIA}

\author{
K. Demova ${ }^{1}$, M. Rosolankova ${ }^{2}$ \\ ${ }^{1}$ Clinic of Neonatology, Faculty hospital, Nove \\ Zamky, Slovakia \\ ${ }^{2}$ Department of Neonatology and Intensive Medicine, \\ Children's faculty hospital, Bratislava, Slovakia
}

Background: Controlled hypothermia improves survival and psychomotor development of newborns with moderate and severe hypoxic-ischemic encephalopathy (HIE).

The aim of registry is to record the patient's data with severe asphyxia systematically, to determine the incidence of perinatal asphyxia and HIE in Slovakia and monitoring the implementation of the whole-body hypothermia in the regions of Slovakia and to follow up the long-term effects, psychomotor development and neuro-sensory disturbances in newborns.

Methods: In 2015, the Committee of Neonatal section of Slovak Pediatric Society approved the project "The National Registry of Perinatal Asphyxia, Hypoxicischemic encephalopathy and Whole-body Hypothermia." The Registry started to collected specific data about newborns with perinatal asphyxia and HIE born in Slovakia and treated with controlled hypothermia. Data collection began from 01.01.2016.
Results: Since the beginning of data collection by the National Registry of Perinatal Asphyxia, Hypoxicischemic encephalopathy and Whole-body Hypothermia 52 neonatology units submitted their reports. On the basis of the data obtained in the Registry (during the period 1.1.31.12.2016) 160 patients were reported. From registered patients $121(75.6 \%)$ had mild HIE, the criteria of Slovak national protocol for controlled whole-body hypothermia fulfilled 39 patients $(24.4 \%)$.

Conclusions: Whole-body hypothermia in newborns after perinatal asphyxia has become standard of care in Slovakia. The expected benefits of registry will be organized and will ensure the possibility of short and long term follow up assessment in neonates with perinatal asphyxia, in infants with HIE without indication of controlled hypothermia and in newborns who were treated with therapeutic hypothermia.

\section{THE SIGNIFICANCE OF NEONATAL CAPILLARY BLOOD PH, BASE DEFICIT AND BICARBONATE LEVELS FOR HYPOXIC ISCHEMIC BRAIN INJURES AND BRAIN BLOOD CIRCULATION CHANGES}

A. Kudreviciene ${ }^{1},{ }^{2}$, A. Basevicius ${ }^{2}$, S. Lukosevicius $^{2}$, J. Laurynaitiene ${ }^{3}$, V. Marmiene ${ }^{4}$, I. Nedzelskiene ${ }^{5}$, R. Tameliene ${ }^{1}$

${ }^{1}$ Lithuanian University of Health Sciences, Department of Neonatology

${ }^{2}$ Lithuanian University of Health Sciences, Department of Radiology

${ }^{3}$ Lithuanian University of Health Sciences, Department of Neurology

${ }^{4}$ Lithuanian University of Health Sciences, Department of Psychiatry

${ }^{5}$ Lithuanian University of Health Sciences, Department of Dental and Oral Diseases

Background: The neonatal capillary blood $\mathrm{pH}$, base deficit (BE) and bicarbonate levels during the first hour of life - one of indicators of perinatal asphyxia. The aim of the study was to evaluate the significance of following for hypoxic ischemic brain injuries in full-term infants and their cerebral blood circulation changes.

Methods: The prospective cross-sectional study was performed at the Department of Neonatology, Lithuanian University of Health Sciences. The studied group consisted of 78 full-term neonates who experienced perinatal asphyxia or hypoxia. During the first hour of life was analyzed the capillary blood $\mathrm{pH}, \mathrm{BE}$ and bicarbonate levels. Ultrasonography and Doppler sonography were conducted during the first five days of life. In order to determine the correlation of cerebral blood flow parameters 
of anterior cerebral arteries with neonates' capillary blood pH, BE, and bicarbonate levels, Pearson's and Spearman's correlation analysis were applied.

Results: $\mathrm{pH}<7.0 \quad(r=0.4 ; p<0.001)$ and $\mathrm{BE} \leq-12$ $\mathrm{mmol} / \mathrm{L}(\mathrm{r}=-0.2 ; \mathrm{p}=0.02)$ was associated with brain swelling and thalamus and/or basal ganglia injury and brainstem injury. $\mathrm{pH}<7.0$ and $\mathrm{BE} \leq-12 \mathrm{mmol} / \mathrm{L}$ were associated with a higher peak systolic velocity (Vs) $(p<0.005)$ and end-diastolic velocity (Vd) $(p<0.005)$. $\mathrm{pH}<7.0$ was associated with a lower resistive index (RI) $(p<0.05)$. We found no significant relationship between pathological bicarbonate data with hypoxic ischemic injuries and cerebral blood flow parameters.

Conclusions: Neonatal capillary blood $\mathrm{pH}$ and $\mathrm{BE}$ correlated with hypoxic ischemic brain injuries detected during ultrasonography and influenced changes in cerebral blood circulation.

\section{IDENTIFICATION OF A PANEL OF CYTOKINES IN INFANTS AT RISK OF BRAIN DAMAGE TREATED WITH HYPOTHERMIA}

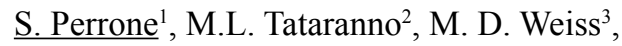 \\ R. Candace ${ }^{3}$, M. Longini ${ }^{1}$, F. Proietti ${ }^{1}$, \\ S. Cornacchione ${ }^{1}$, F. Bazzini ${ }^{1}$, E. Belvisi ${ }^{1}$, \\ G. Buonocore ${ }^{1}$
}

\section{${ }^{1}$ Department of Molecular and Developmental Medicine, General Hospital "Santa Maria alle Scotte”, University of Siena, Italy ${ }^{2}$ Department of Neonatology, Wilhelmina Children's Hospital, University Medical Centre Utrecht, Utrecht, The Netherlands ${ }^{3}$ Department of Pediatrics, University of Florida, Gainesville, FL, USA}

Background: Therapeutic hypothermia (TH) improve neurological outcome in asphyxiated term newborns. Currently, little is known about the impact of hypothermia on the inflammatory response triggered by hypoxia-ischemia in neonates with hypoxic ischemic encephalopathy (HIE). The aim of our study was to identify a panel of cytokines involved in brain injury in neonates with HIE and possibly modulated by $\mathrm{TH}$.

Methods: Forty-three newborns were enrolled: 35 presented HIE and were subjected to TH (HIE Group), 8 were healthy (CTRL Group). For the HIE group, a total of 5 samples were collected: between 0 and 6 hours of life (time 1), to 12 hours (time 2), 24 hours (time 3), 48 hours (time 4) and 72 hours of life (time 5). For CTRL group one sample (time 1) was collected. A panel of 48 inflammatory cytokines was determined in all samples. Data were analyzed using multivariate statistical analysis (Principal Component Analysis-PCA).
Results: 17 cytokines, among 48 analyzed, were found to be significantly different, initially, between the CTRL and HIE groups: 12 with reported pro-inflammatory effects and 5 with reported anti-inflammatory effects. In HIE group cytokine values were significantly higher at time 1 than time 5, showing a decreasing trend during the $\mathrm{TH}$ and at the end of treatment were comparable to the CTRL group.

Conclusions: Hypothermia modulates inflammatorysensitized hypoxia-ischemia insult. The identification of 17 cytokines as biomarkers of perinatal asphyxia and targets of neuroprotective TH provides a useful tool for identifying new personalized strategies in the field of precision medicine.

\section{EARLY BRAIN FMRI CHANGES AFTER PERINATAL HYPOXIA-ISCHEMIA}

S. Kerrn-Jespersen ${ }^{1}$, T.B. Henriksen ${ }^{1}$, H. Brogaard ${ }^{1}$, T.C. Andelius ${ }^{1}$, M. Pedersen ${ }^{2}$, K.J. Kyng ${ }^{1}$

${ }^{1}$ Perinatal Research Unit, Department of Clinical Medicine, Aarhus University Hospital, Denmark ${ }^{2}$ Comparative Medicine Lab, Department of Clinical Medicine, Aarhus University Hospital, Denmark

Background: Perinatal hypoxia-ischemia is a major cause of brain injury and death in term babies. Functional magnetic resonance imaging (fMRI) is a useful biomarker for injury, clinically and in therapeutic trials. However, the optimal timing of fMRI is unknown. The aim of this study was to evaluate cerebral changes after hypoxia-ischemia by serial fMRI studies in a piglet model.

Methods: Six piglets (mean age $19.5 \mathrm{~h} ; 1533 \mathrm{~g}$ ) were anesthetized and mechanically ventilated. $\mathrm{F}_{\mathrm{i}} \mathrm{O}_{2}$ was lowered to $4 \%$ for 45 minutes to achieve global hypoxia and hypotension, then adjusted to maintain EEG $<7 \mu \mathrm{V}$. fMRI (3 Tesla) was performed before (baseline), and 2, 6 , 12 and 18 hours after hypoxia-ischemia. Apparent diffusion coefficient (ADC: a predictor for edema), relative cerebral perfusion $(\mathrm{rCBF})$ and Lactate $/ \mathrm{N}$-acetyl aspartate (Lac/NAA)-ratio were analyzed in the basal ganglia, thalamus and white matter.

Results: In 3 piglets that died during the experiment ADC decreased 50\% from baseline to $6 \mathrm{~h}(p=0.08)$. ADC was not significantly changed in the piglets that survived throughout the experiment. Lac/NAA-ratio increased in all piglets, with a peak $6-12 \mathrm{~h}$ after baseline, depending on brain region ( $p<0.05$ in white matter). Lac/NAA-ratio was highest in piglets with low ADC. A non-significant $6 \%$ increase in $\mathrm{rCBF}$ was seen from baseline to $6 \mathrm{~h}$.

Conclusion: The dynamic early ADC, Lac/NAA-ratio and $\mathrm{rCBF}$ changes suggest an optimal timing of fMRI at $6-12 \mathrm{~h}$ in this model. The results contribute to our understanding of how hypoxic-ischemic brain injury evolves, and have implications for the design of future therapeutical trials using fMRI as an outcome. 


\section{IMPACT OF THE HYPOXIC-ISCHEMIC ENCEPHALOPATHY SEVERITY IN RESPONSE TO CARDIOVASCULAR REGULATION DURING THERAPEUTIC HYPOTHERMIA AND REWARMING}

\author{
M. Kozar ${ }^{1}$, K. Javorka ${ }^{2,3}$, M. Mestanik ${ }^{2}$, \\ K. Matasova ${ }^{1}$, M. Zibolen ${ }^{1}$
}

${ }^{1}$ Clinic of Neonatology, ${ }^{2}$ Martin Biomedical Centre and ${ }^{3}$ Department of Physiology, Jessenius Medical Faculty in Martin, Comenius University in Bratislava, University Hospital in Martin, Slovakia

Background: Whole body hypothermia can reduce the consequences of hypoxic - ischemic encephalopathy (HIE) in asphyctic newborns. Aim of the study was to identify impact of posthypoxic brain injury on cardiovascular regulation in response to therapeutical hypothermia and body temperature recovery in asphyctic newborns.

Methods: Eight asphyctic term neonates were enrolled into the study. Following parameters were recorded during hypothermia $\left(33.5^{\circ} \mathrm{C}\right)$ and rewarming to normothermia $\left(36.5^{\circ} \mathrm{C}\right)$ : RR intervals, heart rate variability (HRV) [lowfrequency power (LF-HRV), high-frequency power (HFHRV)], blood pressure (BP) and oxygenation parameters [blood oxygen saturation $\left(\mathrm{SpO}_{2}\right)$, cerebral $\left(\mathrm{rcSO}_{2}\right)$ and splanchnic tissue oxygenation $\left.\left(\mathrm{rsSO}_{2}\right)\right]$. Patients were divided into two groups according to the severity of hypoxic - ischemic encephalopathy [moderate $(n=4)$, severe $(n=4)$ ].

Results: Significant rise in mean HR $(p<0.05)$ together with elevation of LF-HRV without change in HF-HRV (index of cardiovagal regulation) and a decrease of BP $(p<0.05)$ were observed during body temperature recovery. Rewarming procedure led to a light fall in $\mathrm{SpO}_{2}$, while $\mathrm{rcSO}_{2}$ was stable. Values of $\mathrm{rsSO}_{2}$ were different between groups depending on the brain injury severity. Subjects with severe HIE showed lower values of the HRV and $\mathrm{BP}$ and higher increase in $\mathrm{HR}$ and $\mathrm{rcSO}_{2}$ during rewarming.

Conclusions: Cardiovascular regulatory mechanisms seem to be affected by severity of HIE in asphyctic newborns.

\section{THROMBOELASTOGRAPHY AND COAGULATION PROFILE IN NEONATES WITH PERINATAL HYPOXIA-ASPHYXIA}

\author{
A. Konstantinidi ${ }^{1}$, R. Sokou ${ }^{1}$, A. Tsantes ${ }^{2}$, \\ S. Valsami ${ }^{3}$, E. Kouskouni ${ }^{4}$, A. Gounaris ${ }^{5}$, \\ $\underline{N}^{\text {. Iacovidou }}{ }^{6}$
}
${ }^{I}$ NICU, “Aghios Panteleimon”, Nikaia General Hospital, Athens, Greece
${ }^{2}$ Blood Bank, "Attikon" University General
Hospital, National and Kapodistrian University of Athens
${ }^{3}$ Haematology Laboratory-Blood Bank, NKUA
Aretaieion Hospital
${ }^{4}$ Biopathology Laboratory, NKUA, Aretaieion
Hospital
${ }^{5}$ NICU, University Hospital of Larisa
${ }^{6}$ Department of Neonatology, NKUA, Aretaieion
Hospital

Background: Perinatal hypoxia-asphyxia causes clotting derangement of the already immature mechanism of hemostasis in the neonate. ThromboelastographyThromboelastometry (TTEG/TEM) evaluates the clotting cycle, from platelet activation to fibrinolysis, providing information for the time spent to clot formation and its stability, and allows prompt intervention. This prospective study aims at evaluating the derangement of hemostasis in neonates with perinatal hypoxia/severe asphyxia with rotational-thromboelastometry ROTEM.

Methods: 81 neonates with perinatal hypoxia/severe asphyxia were studied. They were subdivided in 2 groups: Group A with 74 neonates with perinatal hypoxia/stress and group B with 7 asphyxiated neonates. ROTEM parameters (CT-clotting time, CFT-clot formation time, MCF- Maximal Clot Firmness) were compared with values in healthy neonates. ANOVA test was used for statistical analysis.

Results: In all neonates of the study mean CT, CFT and MCF, was $51.83 \mathrm{sec}, 94 \mathrm{sec}$ and $62.7 \mathrm{~mm}$, respectively. In controls mean CT, CFT and MCF was $44.4 \mathrm{sec}, 78.4 \mathrm{sec}$ and $65.64 \mathrm{~mm}$, respectively. Mean values CT and CFT in study population vs that in controls are significantly different $(p<0.05)$. In group A mean CT, CFT and MCF was $48.1 \mathrm{sec}, 83.8 \mathrm{sec}$ and $63.3 \mathrm{~mm}$, respectively. In group B mean CT, CFT and MCF was $91.4 \mathrm{sec}, 129.5 \mathrm{sec}$ and $56.7 \mathrm{~mm}$, respectively. These values are significantly different $v s$ group A $(p<0.05)$.

Conclusions: In neonates with perinatal hypoxia/ asphyxia CT and CFT are prolonged vs controls. Clotting derangement is greater in neonates with asphyxia vs hypoxia. TEM could be used for assessment of the degree of this derangement, for prognosis of the risk of hemorrhage and as a guide for relevant interventions in this high-risk group of neonates. 


\section{INTERMITTENT HYPOXIA AUGMENTS PHARMACODYNAMIC RENAL ACTIONS OF CAFFEINE ON PROSTANOIDS AND RECEPTORS IN NEONATAL RATS}

\author{
Aranda JV, Beharry KD
}

\section{State University of New York Downstate Medical} Center, Brooklyn, NY, USA

Background: Intermittent hypoxia (IH) or apnea occurs in all babies $<32$ weeks gestation and may affect drug action and clearance. Caffeine exerts diuresis but its effects on vasoactive mediators in neonatal kidneys remains unclear. We tested the hypothesis that IH amplifies dose-dependent effects of caffeine on neonatal renal vasoactive mediators.

Methods: Newborn rats, randomized to room air (RA), hyperoxia $\left(50 \% \mathrm{O}_{2}\right)$, or $\mathrm{IH}\left(50 \% \mathrm{O}_{2}+\right.$ brief hypoxia, $10 \%$ $\mathrm{O}_{2}$ ) from P0 to P14 were given: 1) standard caffeine doses (SCD) $20 \mathrm{mg} / \mathrm{kg} \mathrm{IP} \mathrm{(loading)} \mathrm{on} \mathrm{P0;} \mathrm{and} 5 \mathrm{mg} / \mathrm{kg} / \mathrm{day}$ IP on P1-P14; 2) high caffeine doses (HiCD) $80 \mathrm{mg} / \mathrm{kg}$ IP on P0 and $20 \mathrm{mg} / \mathrm{kg}$ IP on P1-P14; or 3) saline. At P14, somatic growth, and renal prostanoid production; histopathology; and expression of cyclooxygenases (COX-1\&2), prostanoid receptors and aquaporin-4 (AQP-4) were examined.

Results: IH and hyperoxia had significant adverse effects on somatic growth. SCD produced no adverse effects on renal morphology, however IH amplified detrimental effects of HiCD which produced the most severe renal injury, including marked necrosis and degeneration of glomeruli and tubular structures. with increased COX-2 and TP; and decreased FP, IP, DP, and AQP-4 expression.

Conclusions: IH potentiates adverse effects of high doses of caffeine on renal prostanoids which are important regulators of renal development and function, suggesting toxicity and avoidance of HiCD in preterms.

\section{THE EFFECTS OF NITROUS OXIDE ON THE FETUS AND NEWBORN}

$\underline{\text { L. Nastase }}^{1,2}$, S. Maria-Stoicescu ${ }^{1,2}$, A.Voichitoiu ${ }^{1,2}$

${ }^{1}$ INSMC “Alessandrescu-Rusescu”, Polizu

Maternity, Bucharest, Romania

${ }_{2}^{2}$ Medical and Pharmacy University "Carol

Davila", Bucharest, Romania

Background: Two challenges related to labor and expulsion remains valid and for 21 st century: promoting birth vaginally and maternal pain management in labor. Nitrous oxide is used more frequently for this purpose with good results and minimal risk. Nitrous oxide effects on the fetus are not yet known. We propose immediate postpartum assessment of newborns whose mothers had received nitrous oxide.
Methods: Study was performed prospectively on a lot of 30 newborns with VG over 36 weeks structured as follows: subgroup 1 consists of 10 newborns whose mothers had received nitrous oxide and subgroup 2 consists of 20 newborns whose mothers received other forms of intrapartum analgesia. Newborns were evaluated clinically (Apgar score), biochemical (acid-base balance from umbilical cord) and paraclinically (cerebral tissue oxygenation in vivo spectroscopy NIRS-INVOS).

Results: Infants had similar clinical evaluation Apgar score for two subgroups. Those whose mothers had received nitrous oxide had slightly increased of $\mathrm{pH}$ blood from umbilical artery, and cerebral saturation was higher in the first 10 minutes of life compared to the comparative group.

Conclusions: Maternal intrapartum analgesia with nitrous oxide may have some beneficial effects on the fetus compared to other methods of analgesia. Further extended studies are needed to confirm the premises above mentioned.

\section{MODE OF DELIVERY INFLUENCES NOCICEPTIVE SENSITIVITY IN NEWBORN INFANTS - THE NOCICOP TRIAL}

$\underline{\text { S. Wellmann }}^{1}$, S. Kasser ${ }^{1}$, C. Hartley ${ }^{2}$, H. Rickenbacher ${ }^{1}$, N. Klarer ${ }^{1}$, A. Depoorter ${ }^{3}$, A.N. Datta ${ }^{3}$, W. Magerl ${ }^{4}$, E.A. Huhn ${ }^{5}$, G. Green ${ }^{2}$, R. Slater ${ }^{2}$

${ }^{1}$ Division of Neonatology, University of Basel Children's Hospital (UKBB), Basel, Switzerland ${ }^{2}$ Department of Pediatrics, University of Oxford, Oxford, UK

${ }^{3}$ Division of Neuropediatrics \& Developmental

Medicine, University of Basel Children's Hospital (UKBB), Basel, Switzerland

${ }^{4}$ Department of Neurophysiology, University of

Heidelberg, Heidelberg, Germany

${ }^{5}$ Department of Obstetrics and Gynecology, University Hospital Basel, Basel, Switzerland

Background: The effect of delivery mode and birth stress on nociception of the newborn infant is largely unknown.

Objective: to determine whether pain-related brain activity differs between vaginally born infants and those born by elective cesarean section (ECS) shortly after birth.

Methods: A prospective, single-center study was performed at the University Hospital of Basel enrolling 41 healthy full-term infants, 22 delivered by spontaneous vaginal delivery (VD) and 19 by ECS. The primary outcome measure was the magnitude of the noxiousevoked brain activity following the application of a mildly noxious experimental stimulus applied to the infant's right 
hand 5 hours after birth. In addition, a surrogate marker of vasopressin, copeptin, was quantified in the umbilical cord blood, and the infant's sex, gestational age, fetal distress during labor, and the length of labor were considered in the analysis.

Results: The magnitude of the noxious-evoked brain activity was significantly greater in infants born by ECS $(0.26 \pm 0.11$, mean \pm SD) compared to infants born by VD $(0.18 \pm 0.07$ CI: $[0.025,0.15], p<0.01)$. Furthermore, the magnitude of the noxious-evoked brain activity was inversely proportional to the amount of fetal copeptin recorded ( $\beta=-0.024$, CI: $[-0.04,-0.004], p=0.019)$, and was significantly greater in VD infants who experienced fetal distress during delivery ( $p=0.029$, CI: $[0.009,0.14])$.

Conclusion: Infants born by VD have lower painrelated brain activity compared with infants born by ECS a few hours after birth, which is related to the degree of fetal vasopressin production and modulated by birth stress.

\section{FENTANYL FOR PROCEDURAL PAIN IN PRETERM NEWBORN INFANTS - A PHARMACOKINETIC AND PHARMACODYNAMIC STUDY}

\author{
V. Fellman $^{1}$, J. Kindblom ${ }^{1}$, A.-C. Berg ${ }^{1}$, C. Halzius ${ }^{3}$, \\ G. Printz ${ }^{3}$, A. Rane ${ }^{3}$, H. Lagercrantz ${ }^{3}$, B. Hallberg ${ }^{3}$, \\ E. Norman ${ }^{1}$ \\ ${ }^{1}$ Pediatrics, Lund University, Lund, Sweden \\ ${ }^{2}$ Inst. Medicine, University of Gothenburg, Sweden \\ ${ }^{3}$ Karolinska Hospital and Karolinska Institutet, \\ Stockholm, Sweden
}

Background: Newborn infants are entitled to pain relief during neonatal intensive care. Opioids are mostly used for both procedural and continuous pain even though they have numerous side effects and are used off-label in this population. As fentanyl has rapid onset and a short action without any active metabolites, we hypothesized that a low -dose prior to a short procedural pain would be sufficient relief in preterm infants.

The aim was to study pharmacokinetics (PK) and pharmacodynamics of a fentanyl investigational newborn formulation $(5 \mathrm{mcg} / \mathrm{ml})$ administered for procedural pain.

Methods: To this Swedish multi-center study (NeoFent-I, EUDRACT 2011-000310-19; Within the NeoOpioid EU- FP 7 GA 223767) 30 preterm infants born at a median gestational age of $26.9+6$ gestational weeks (range 26.3+2-34.0+1) with birth weight of $852 \mathrm{~g} \mathrm{(530}$ $-1706 \mathrm{~g}$ ) who had not received opioids the last $72 \mathrm{~h}$ were included at a postnatal age of 6.9 days of age $(0.25-28.2$ days) in a Swedish multicenter study, the NeoFent-I study. They received $0.5 \mu \mathrm{g} / \mathrm{kg}$ fentanyl i.v. (to be repeated if needed) before insertion of a peripheral cannula or $2 \mathrm{mcg} /$ $\mathrm{kg}$ before intubation. Blood samples for PK analyses were obtained at $10-30 \mathrm{~min}, 2,4,8$ and $24 \mathrm{~h}$ after drug administration. The procedural pain was assessed with BIIP and PIPP-R and the continuous pain/stress before and every hour after the procedure with ALPS-Neo.

Results: PK results (infants with at least 3 measured concentrations):

\begin{tabular}{|l|c|c|c|c|}
\hline Variable & \multicolumn{2}{|l|}{$\begin{array}{l}\text { Dose 1 }(0.5 \mathrm{mcg} / \mathrm{kg}) \\
n=9\end{array}$} & \multicolumn{2}{l|}{$\begin{array}{l}\text { Dose 2 } \\
n=5\end{array}$} \\
\hline & Mean (SD) & Range & Mean (SD) & Range \\
\hline Half time, $\mathrm{h}$ & $12.5(6.64)$ & $1.6-20.5$ & $16.0(13.5)$ & $4.1-32.6$ \\
\hline $\mathrm{C} 0, \mathrm{ng} / \mathrm{mL}$ & $0.29(0.26)$ & $0.17-0.98$ & $0.47(0.09)$ & $0.32-0.57$ \\
\hline $\begin{array}{l}\text { Volume of } \\
\text { distribution, } \\
\mathrm{L} / \mathrm{kg}\end{array}$ & $2.45(0.87)$ & $0.52-3.21$ & $4.5(1.2)$ & $3.5-6.7$ \\
\hline $\begin{array}{l}\text { Clearance, } \\
\mathrm{L} / \mathrm{kg} / \mathrm{h}\end{array}$ & $0.16(0.07)$ & $0.11-0.30$ & $0.36(0.28)$ & $0.09-0.71$ \\
\hline
\end{tabular}

The 10 min fentanyl concentration did not correlate with the pain scores. In the low-dose group, a concentration $>0.15 \mathrm{ng} / \mathrm{mL}(n=11)$ was associated with no/low pain in $73 \%$ and $64 \%$ using BIIP (cutoff limit $<4$ ) and PIIP-R $(<8)$ scores, respectively.

Conclusions: Fentanyl $0.5 \mathrm{mcg} / \mathrm{kg}$ did not ameliorate pain in all cases but the pain reaction was not severe enough for clinical indication to repeat the dose. As the half-time and clearance values are very variable factors influencing fentanyl metabolism need to be clarified before dose recommendations can be defined for preterm infants.

\section{NEURODEVELOPMENTAL OUTCOME IN VERY PRETERM INFANTS RANDOMIZED TO RECEIVE TWO DIFFERENT PARENTERAL NUTRITION REGIMENS: THE SCAMP NUTRITION STUDY}

\author{
$\underline{\text { C Morgan }}^{1}$, S Parry $^{1}$, M Tan $^{2}$ \\ ${ }^{1}$ Liverpool Women's Hospital, Liverpool, UK \\ ${ }^{2}$ Alder Hey Children's Hospital, Liverpool, UK
}

Background: Increasing parenteral protein and energy intake using a Standardized, Concentrated with Added Macronutrients Parenteral (SCAMP) nutrition regimen ameliorates early head growth failure in very preterm infants (VPI). Aim of the study was to compare neurodevelopmental outcome at 2-3 years in VPI randomized to receive SCAMP nutrition or control regimen.

Methods: VPI were originally randomized to either start SCAMP or remain on the control regimen before day 5. The consent process included neurodevelopmental assessment (Bayley III) all performed (blinded) by coauthors MT or SP between 2 and 3.5 years corrected gestational age. The raw scores of each subtest were converted to scaled and composite scores. 


\begin{tabular}{|l|l|l|l|l|l|}
\hline Group (n) & Age (mths) & Cognitive & Language & Motor & Combined \\
\hline SCAMP: All infants (38) & $29.2(3.7)$ & $87(15)$ & $81(18)$ & $79(13)$ & $84(15)$ \\
\hline Control: All infants (41) & $30.0(3.9)$ & $81(14)$ & $76(17)$ & $76(15)$ & $78(14)$ \\
\hline p-value & $\mathbf{0 . 4 9}$ & $\mathbf{0 . 0 8}$ & $\mathbf{0 . 1 1}$ & $\mathbf{0 . 3 8}$ & $\mathbf{0 . 0 9}$ \\
\hline SCAMP: 24-26 wks (15) & $28.9(3.4)$ & $85(17)$ & $75(20)$ & $74(15)$ & $80(18)$ \\
\hline Control: 24-26 wks (16) & $30.3(3.5)$ & $75(16)$ & $67(13)$ & $69(18)$ & $71(13)$ \\
\hline p-value & $\mathbf{0 . 3 2}$ & $\mathbf{0 . 1 1}$ & $\mathbf{0 . 2 5}$ & $\mathbf{0 . 4 4}$ & $\mathbf{0 . 1 4}$ \\
\hline SCAMP: score 50-85 (\%) & $29.2(3.7)$ & $11(29)$ & $16(42)$ & $21(55)$ & $11(29)$ \\
\hline Control: score 50-85 (\%) & $30.0(3.9)$ & $18(47)$ & $29(71)$ & $27(66)$ & $21(51)$ \\
\hline p-value & $\mathbf{0 . 4 9}$ & $\mathbf{0 . 1 7}$ & $\mathbf{0 . 0 1 3 *}$ & $\mathbf{0 . 3 6}$ & $\mathbf{0 . 0 7}$ \\
\hline
\end{tabular}

Results: The composite score data are summarized below as mean (sd) Bayley III composite scores in SCAMP versus control groups.

Conclusion: This study suggests benefits for neurodevelopmental outcome with the SCAMP regimen consistent with previous improvement in early head growth.

\section{IMPACT OF EARLY AGGRESSIVE NUTRITION ON VERY LOW BIRTH WEIGHT INFANTS ON WEIGHT, LENGTH AND HEAD CIRCUMFERENCE. A RETROSPECTIVE STUDY}

\author{
R. Hentschel ${ }^{1}$, A. Homburg ${ }^{1}$, P. Franck ${ }^{1}$, M. Kunze ${ }^{2}$ \\ ${ }^{1}$ Dept. of General Pediatrics, Div. Neonatology/ \\ Intensive Care Medicine, Medical Center, \\ University of Freiburg, Germany \\ ${ }^{2}$ Dept. of Obstetrics and Gynecology, Medical \\ Center, University of Freiburg, Germany
}

Introduction: The concept of early and "aggressive" nutrition (AN) of very low birth weight (VLBW) infants, introduced by Thureen and Ziegler about 15 years ago, is nowadays standard of care.

Aim was to study a potential differential impact of AN on growth charts for weight, length and head circumference in a cohort of all VLBW infants born in 2013 with AN vs. a cohort from 2002 without AN.

Methods: Retrospective analysis from patients' records from birth till day 50 in a single center. Recording of (1) anthropometric data, according to percentiles from Voigt (2006) and transformation to z-score differences, and (2) detailed nutritional intake data (energy, parenteral vs. enteral nutrition, glucose, protein, fat and others). Statistics: t test, Mann-Whitney test, Fisher's exact test, as appropriate. Significance assumed for $\mathrm{p}<0.05$.

Results: 44 infants from 2013 and 42 from 2002 were eligible. Gestational age (median $28+1 / 7$ vs. $26+6 / 7$ ), weight, and head circumference were significantly higher in 2013 vs. 2002. However, z-scores for weight length and head circumference at birth were not different. Even though duration of treatment was shorter in $2013(p=0.002)$ courses of z-scores for weight $(p=0.006)$ and length $(p=0.0001)$, but not for head circumference $(p=0.213)$, were significantly better in 2013 vs. 2002. In a multiple linear regression analysis using two different models either total accumulated energy, or total protein, total fat and SGA status were the most important determinants for adequate weight gain from day 5 to day 30 .

Conclusion: There might be a differential effect of AN on the anthropometric measures with a weaker effect on head circumference.

\section{VITAMIN D STATUS AND ADEQUACY OF STANDARD SUPPLEMENTATION IN VLBW INFANTS}

St. Hitrova-Nikolova $^{1}$, B. Slancheva ${ }^{1}$, N. Jekova ${ }^{1}$, A. Popivanova ${ }^{1}$, L. Vakrilova ${ }^{1}$, P. Radulova ${ }^{1}$, N. Yarakova ${ }^{1}$, T. Pramatarova ${ }^{1}$, Z. Emilova ${ }^{1}$

${ }^{1}$ Department of Neonatology, University hospital of Obstetrics and Gynecology "Maichin dom" Chair of Obstetrics and Gynecology, Faculty of Medicine at Medical University of Sofia, Bulgaria

Background: The aim of our study was to examine the 25-hydroxy vitamin D (25-OHD) status and biochemical bone indices of very low birth weight (VLBW) infants at birth and at 8 weeks of life and to evaluate the adequacy of the standard 25-OHD supplementation.

Methods: This prospective cohort study enrolled 41 preterm infants $<32$ weeks of gestation with birth weight $<1500$ grams treated at a tertiary neonatal center. The levels of 25-OHD at birth were measured in venous cord blood by test analysis "ECLIA". All infants were fed with preterm baby formula, fortified breast milk and were given vitamin D3 1334 IU / per day from day 20. Serum 25-OHD was measured again at 8 weeks of life. Biochemical indicators of bone metabolism/ionized calcium, phosphorus, alkaline phosphatase were estimated in two week intervals till eight weeks of age. Parathormon (PTH) levels were measured twice at 4 and 8 weeks of age.

Results: Using a cutoff value of $<20 \mathrm{ng} / \mathrm{ml}$ to determine deficiency and $<30 \mathrm{ng} / \mathrm{ml}$ insufficiency we found that $32 \%$ 
of the babies were 25-OHD insufficient and 49\% were deficient at birth. At eight weeks of age $70 \%$ of the infants had sufficient levels of vitamin D and $30 \%$ of the children remained deficient despite the recommended supplementation with vitamin D. Statistically significant negative linear relationship was observed in the dynamics of the PTH levels and vitamin D $(p<0.0001)$. Other biochemical markers of bone metabolism were in reference limits.

Conclusions: The prevalence of vitamin D insufficiency in VLBW infants is high at birth. However a high percentage of them remain insufficient at 8 weeks of age despite they were supplemented with a dose of 1334 IU/ day starting from age of 20 days. The recommended time of beginning of the supplementation and the dose of vitamin D 1334 IU/day appears to be inadequate to prevent vitamin D insufficiency. Further randomized controlled trials with lager sample of patients are needed to determine the optimal dosage of 25-OHD supplementation in VLBW infants.

\section{QUANTITATIVE ULTRASOUND ASSESSMENT OF BONE HEALTH IN THE NEWBORNS}

\author{
A. Soltirovska-Salamon ${ }^{1}$, J. Salobir ${ }^{1}$, \\ D. Paro-Panjan ${ }^{1}$ \\ ${ }^{1}$ University Medical Centre Ljubljana, Division of \\ Pediatrics, Department of neonatology, Slovenia
}

Background: Bone mineralization is one of the major challenges of management in newborns with risk factors for bone development; osteopenia is recognized with increasing frequency. Our study aimed to evaluate the feasibility of quantitative ultrasound (QUS) in evaluation of newborn's bone health.

Methods: QUS evaluation was performed at the right tibia by measuring two parameters: speed of sound (SOS, in $\mathrm{m} / \mathrm{s}$ ) and as standard deviation relative to normal controls (Z-score). QUS parameters were related to the anthropometric characteristics, risk factors for bone development and biochemical parameters.

Results: Analysis of variance in 181 newborns (average age 26 days) with gestational age between 23 and 42 weeks (mean $33.8 \pm 5.0$ ) and a birth weight from 405 to $5130 \mathrm{~g}$ (mean $2132 \pm 1091 \mathrm{~g}$ ) shows significantly lower QUS values in preterms ( $\mathrm{SOS}, p=0.003$; Z-score, $\mathrm{p}=0.05$ ). Regression analysis shows correlation between QUS measurement and birth anthropometry: birth weight, birth length and head circumference (SOS, $\mathrm{p}<0.001$; Z-score, $\mathrm{p}<0.001)$. QUS values correlated significantly with serum concentration of vitamin D (VD) (SOS, $r=0.356$; $p=0.013 ;$ Z-score, $r=-0.558 ; \quad \mathrm{p}<0.001)$ and urinary concentration of phosphate/creatinine ratio (UrP/creat) (SOS $r=-0,431 ; p=0,003 ; Z$-score $r=-0,536, \mathrm{p}<0,001$ ).
Regression analysis demonstrated a statistically significant link between VD and UrP/creat $(\mathrm{r}=-0.374 ; p=0.01)$.

Conclusions: Our results demonstrate that QUS measurements are related to risk factors and biochemical parameters and therefore QUS is a reliable method for assessment of bone health.

\section{RADIATION EXPOSURE IN VERY LOW BIRTH WEIGHT INFANTS}

F.C.C.M. Pinto ${ }^{1}$, G. Rocha² ${ }^{2}$ F. Flôr-de-Lima ${ }^{2}$, H.E. Soares $^{2}$, I.M.A.P. Ramos ${ }^{3}, \underline{\text { H. Guimarães }}{ }^{2}$

${ }^{1}$ Faculty of Medicine of Porto University, Porto, Portugal

${ }^{2}$ Neonatal Intensive Care Unit, Department of Pediatrics, Centro Hospitalar São João and Faculty of Medicine of Porto University, Porto, Portugal ${ }^{3}$ Department of Radiology, Centro Hospitalar São João and Faculty of Medicine of Porto University, Porto, Portugal

Background: The survival of very low birth weight (VLBW) infants increased in the past few decades. These neonates often require multiple diagnostic and management image procedures that involve ionizing radiation, which can have long-term implications. The aim of our study was to evaluate the level of radiation exposure in VLBW infants during their stay in the neonatal intensive care unit (NICU).

Methods: We collected demographic and medical data of 149 VLBW who were admitted to our NICU between January 2011 and December 2014. All radiographs were review retrospectively. Absorbed ionizing radiation was calculated according to literature reference values.

Results: A total of 1496 radiographic procedures were obtained. Infants received an average of 9.9 radiographs and the maximum of images registered per patient was 64 . Three babies (2\%) had more than 50 radiographs and received more than $1000 \mu \mathrm{Sv}$, the recommended maximum of ionizing radiation exposure. Infants of lower birth weight, who needed invasive ventilation, with bronchopulmonary dysplasia, sepsis, and surgical pathology required significantly more radiographs $(\mathrm{p}<0.001)$.

Conclusions: During their NICU stay, severely ill VLBW were the ones who required more frequent radiological evaluation. It is imperative to minimize radiation exposure through the optimization of the equipment and radiation protection technique. Guidelines for radiation exposure in VLBW must be implemented in all NICU. 


\section{TURKISH NATIONAL MULTICENTRIC ONLINE REGISTRY DATA FOR PREVENTION OF JAUNDICE ASSOCIATED COMPLICATIONS}

\author{
O. Erdeve ${ }^{1}$, E. Okulu ${ }^{1}$, Esin Koc ${ }^{2}$, Turkish Jaundice \\ Registry Data Collabolators ${ }^{3}$ \\ ${ }^{1}$ Department of Pediatrics, Division of Neonatology, \\ Ankara University School of Medicine Children's \\ Hospital, Ankara, Turkey \\ ${ }^{2}$ Gazi University, Ankara, Turkey \\ ${ }^{3}$ Turkey, Turey, Turkey
}

Background: Although jaundice is a common hospitalization reason in the neonatal intensive care unit (NICU), its incidence and complication rates are unknown in Turkey. We conducted an online registry database to determine the risk factors in infants who were hospitalized for jaundice, and tried to define the rate of complications due to acute bilirubin encephalopathy and kernicterus.

Methods: A multi-centric prospective study in infants born $\geq 35$ weeks' gestation and hospitalized for jaundice was conducted between 2015 and 2016. The online records of 5620 patients from 50 collaborator NICUs all around the country for 12 months were evaluated.

Results: The mean gestational age of patients and birth weight were $38 \pm 1.6$ weeks and $3102 \pm 517 \mathrm{~g}$, respectively. The history of siblings treated for jaundice was present in $3.4 \%$ of patients. The leading etiological entities were hemolytic jaundice $(28.9 \%)$, dehydration $(27.6 \%)$, breast milk jaundice (12.6\%) and prematurity $(8.5 \%)$. ABO blood incompatibility was the most common cause (73.7\%) of the hemolytic jaundice. Two-hundred-sixtynine infants $(4.8 \%)$ received intravenous immunoglobulin in addition to phototherapy. Exchange transfusion was performed to $132(2.3 \%)$ patients. Patients who had exchange transfusion had higher rate of hemolytic jaundice, higher reticulocyte count, longer duration of phototherapy and higher bilirubin/albumin ratio $(p<0.05)$. Logistic regression analysis demonstrated that having reticulocyte count $\geq 7$ (OR: 8.83, 95\% $\mathrm{Cl} 3.57-21.87$, $p=0.001$ ) and bilirubin/albumin ratio $\geq 6.5$ (OR: 21.1, $95 \% \mathrm{Cl} 9.6-46.3, p=0.001)$ increased the exchange transfusion rate significantly. Kernicterus was reported in 14 patients $(2.5 \%)$. Male $(85.7 \%)$ gender, hemolytic jaundice, higher reticulocyte count $(\geq 7)$ and bilirubin/ albumin ratio $(\geq 6.5)$ were the major risk factors for the kernicterus $(p<0.05)$.Hearing loss and neurological deficiency were detected as complications in $0.35 \%$ and $0.25 \%$, respectively.

Conclusions: Neonatal jaundice is still a common cause of hospitalization in the NICUs. Dehydration related to lack of proper feeding is one of the most common etiological factors after hemolytic jaundice. Kernicterus and neurological complications reported in our study show that jaundice is still an important public health problem among newborns, and more concentration is needed to prevent its complications.

ISOLATED FETAL ASCITES: PRENATAL AND POSTNATAL MULTIDISCIPLINARY APPROACH

\author{
J. Martic $^{1}$, R. Farrugia ${ }^{1}$, P. Soler ${ }^{1}$, R. Parascandalo ${ }^{1}$ \\ ${ }^{1}$ Neonatal Intensive Care Unit, Mater Dei Hospital, \\ Malta
}

Introduction: Isolated fetal ascites is a rare condition that can be defined as an ascites not associated with hydrops fetalis. A review of literature has shown that it might be idiopathic, caused by genetic and metabolic disorders, congenital infections, chest and intra-abdominal disorders. In view of heterogeneous underlying causes isolated fetal ascites requires a multidisciplinary approach.

Case Report: We report a female baby born at $34+6$ weeks of gestation with a massive isolated ascites. Ascites was first seen on antenatal scan at $31+4$ weeks of gestation. The baby was born in poor condition and required an urgent peritoneal drain insertion in the first hour of life. 923 $\mathrm{ml}$ of serosanguinous and bilious fluid was removed from the abdomen. Ultrasound and MR of the abdomen were performed postnatally showed severe hepatomegaly with no other abnormalities. In view of the bilious drain losses and aspirated air from the drain a laparotomy was done and malrotation and volvulus of mid-ileum with perforation were found. Profuse peritoneal bilious contamination with flecks calcifications imply an antenatal perforation. The child is now 6 months old and asymptomatic.

Conclusions: A careful search for underlying causes should be undertaken in all cases of isolated fetal ascites, preferably antenatally. Antenatal and postnatal treatment is determined by cause and severity of disease and requires multidisciplinary approach and team work.

\section{WHAT DRIVES CHANGE IN NEONATAL INTENSIVE CARE UNITS? RESULTS OF THE QUALITATIVE EPICE STUDY WITH NEONATAL PHYSICIANS AND NURSES IN SIX EUROPEAN COUNTRIES}

M. Cuttini ${ }^{1}$, E. Forcella ${ }^{1}$, and J. Zeitlin ${ }^{2}$, for the EPICE Qualitative Study Group (*)

${ }^{1}$ Pediatric Hospital Bambino Gesù, IRCCS, Roma, Italy

\footnotetext{
${ }^{(*)}$ M. Bonet, A. Lainé, J. Zeitlin (France); R. Meier (Germany); M. Cuttini, E. Forcella (Italy); A. Hasselager (Denmark); E. Draper, J. Willars (UK); A.Martins, C. Rodrigues (Portugal).
} 
${ }^{2}$ Inserm UMR 1153, Obstetrical, Perinatal and Pediatric Epidemiology Research Team (Epopé), Center for Epidemiology and Statistics Sorbonne Paris Cité, DHU Risks in pregnancy, Paris Descartes University, Paris, France

Background: Understanding what drives change in healthcare organizations, and the relationship between triggers for change and successful implementation, is needed for the promotion of evidence-based health care. As part of a mixed-methods international project (EPICE) on the use of effective interventions for the care of very preterm infants, we carried out a qualitative study to explore how clinical or organizational changes are introduced and implemented in the neonatal intensive care unit (NICU), and what may facilitate or hinder the process.

Methods: We carried out individual semi-structured interviews with the medical and nursing staff of 11 randomly selected NICUs in six European regions, focusing on the last clinical or organizational change introduced in the unit. Overall, 44 interviews were carried out, anonymously recorded and transcribed verbatim. Thematic analysis was performed using NVivo 10 software.

Results: Five main triggers for change ("themes") were identified: 1) the need to standardize practices; 2) the adaptation of newly issued guidelines; 3 ) new scientific information from the literature, scientific meetings or contacts with colleagues; 4) attempts to improve patient care; and 5) other reasons, such as participation in a multicentric study requiring modification of the unit's routines, or a severe adverse event leading to patient harm. Of these triggers, perceived benefit to patients through improved care was also reported as a facilitator, linked to staff compliance and successful implementation.

Conclusions: Clinical and organizational innovations that are initiated to improve the patients' outcomes and wellbeing are more likely to achieve consensus and lead to sustained change.

\section{IMPLEMENTATION OF FIGO MOTHER- FRIENDLY HOSPITAL INITIATIVE IN CROATIA - ROLE OF NEONATOLOGIST}

\section{$\underline{\text { M. Stanojevic }}^{1}$ \\ ${ }^{1}$ Department of Obstetrics and Gynecology Medical School University in Zagreb, Neonatal Unit, Zagreb, Croatia}

Background: All (30) public maternity wards (MW) in Croatia are Baby Friendly (BF) while none is Mother Friendly (MF). FIGO guidelines launched in 2015 prompted four MW to implement them with help of the Ministry of Health and UNICEF Office in Croatia.
Aim of the paper was to present the obstacles during the implementation of MF initiative using experience from the implementation (mostly by neonatologists) of BF initiative.

Methods: FIGO guidelines have been used to produce core documents for the implementation of MF initiative including three leading principles, ten steps of MF, selfappraisal, and external monitoring tools. All documents have been written using the same methodology as in BF initiative documents, based on FIGO paper on MF. Four MW agreed on to participate in the pilot project.

Results: In every MW participating in the pilot the following steps have been undertaken: establishment of the implementation team, education of educators for the implementation of MF, education of the personnel, standardization of the procedures, writing the information for staff and patients. The most serious obstacle was to agree on the standardization of procedures in all MW.

Conclusions: It has been planned to finish the pilot after 6 months (April-October, 2017) with external evaluation and thorough analysis of implemented steps. Then the possibility to spread out the initiative to all MW in Croatia will be explored.

\section{KANGAROO CARE, A STRATEGY FOR DEVELOPMENT - CENTERED CARE}

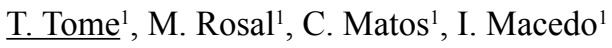 \\ ${ }^{1}$ Women's and Children Center, Hospital Center, \\ Neonatal Department Lisbon, Portugal
}

Background: Nowadays the philosophy of Neonatal Intensive Care Units (NICUs) is based in the quality of survival. Thus, exclusively medicalized care has been replaced by family centered care. Skin to skin contact, Kangaroo care $(\mathrm{KC})$, has been progressively expanded in developed countries and used as a transversal strategy in a model (Quantum Caring).

Our aim was to evaluate the effect of a training project on staff and parents and short-term outcomes in preterm infants submitted to KC in a level 3 NICU.

Methods: Implementation of a pilot project with impact evaluation by a survey in parents and staff. Case control study in which the case group received $\mathrm{KC}$, compared with a historic group adjusted for gestational age. We analyzed length of stay, breast-feeding at discharge, weight gain, severe intraventricular hemorrhage and late sepsis.

Results: After the implementation process we found an increase in knowledge and greater use of $\mathrm{KC}$ by parents and staff. In the study group we found better weight gain and greater success of breastfeeding.

Conclusions: A process of dynamization of training and implementation leads to greater acceptance of this practice. In regard to short term outcome we find benefits in preterm infants but medium and long term evaluation is needed. 
THE EFFECT OF THE CLOSE COLLABORATION WITH PARENTS TRAINING FOR THE NEONATAL INTENSIVE CARE UNIT STAFF ON PARENTS' DEPRESSION UP TO 2 YEARS AFTER A PRETERM

$\underline{\text { L. Lehtonen }}^{1}$, S. Ahlqvist-Björkroth ${ }^{1}$, A. Axelin ${ }^{1}$, Z. Boukydis ${ }^{1}$

${ }^{1}$ University of Turku, Finland, Turku University Hospital, Turku, Finland

Background: Training programs for neonatal intensive care unit staff such as the Close Collaboration with Parents (CC) Training ${ }^{\mathrm{TM}}$ may improve psychological wellbeing of parents of preterm infants. This study evaluated the effects of the CC Training ${ }^{\mathrm{TM}}$ on parents' depression.

Methods: Depression was screened among the parents of infants $\leq 1500 \mathrm{~g}$ as a part of the PIPARI Study (www. utu.fi/pipari) in 2001-2006. After the CC Training in 2009-2010, the post-intervention cohort was collected in 2011-2015. The mothers were asked to fill in the Edinburgh Postnatal Depression Scale (EPDS) at 4 and 6 months of corrected age, and both parents were asked to fill in the Beck Depression Inventory at 2 years of corrected age of the child. The analyses were adjusted for mothers' previous mental health problems.

Results: The EPDS was returned by 145 mothers at baseline and by 119 mothers after intervention. The adjusted EPDS scores were 2.79 points lower $(p<0.0001)$ in the post-intervention cohort compared to the baseline. The post-intervention group had lower depression scores consistently over gestational age groups but the effect size was largest in infants born at 28 to 31 gestational weeks. The percentage of mothers with EPDS scores above 12 decreased from $10 \%$ to $2 \%$. At 2 years of age, $17 \%$ of the mothers and $13 \%$ of the fathers exceeded the threshold for depression at baseline. The post-intervention data of the 2-year measurements will be presented in the workshop.

Conclusions: The CC Training seemed to have longlasting positive effects on parents' psychological wellbeing. Parents' well-being is likely to be reflected in the child development later.

\section{TREATMENT OF EXTREMELY PREMATURE INFANTS - A BALANCE BETWEEN ETHICS AND PRIORITIES}

D. Bratlid ${ }^{1}$

\section{${ }^{1}$ Department of health administration and health economics, University of Oslo, Norway}

Background: Developments in treatment have continuously improved survival and reduced morbidities in pre- mature infants. However, during the last decades increases in survival and reduction in morbidities have been marginal although treatment of extremely premature infants now is the most expensive in-hospital patient treatment. Human and economic costs and morbidities now exceed the benefit of infants surviving with a good quality of life. The concept of the grateful dead reflects this.

Focus on technology and limits for treatment: An increased focus on further technology improvements such as ESPI technique (Extracorporal Support of the Premature Infant) have been suggested to increase survival and reduce morbidity of these infants. Another possibility could be to define a gestational age (GA) limit for active treatment. However, when the 26 GA week limit in The Netherlands was reduced to 25 in 2006, both survival as well as morbidities in survivors increased.

Ethics and priorities: New technologies and limits for active treatment raise ethical and priority questions. Treatment limits for groups of patients are rare for other diagnoses and could be unethical. Treatment priorities are usually also based on individual evaluation. However, such individual evaluation of treatment strategies has unfortunately resulted in significant differences between hospitals.

The (individual) balance: Treatment limits based on GA would leave many parents without a vote, and do not consider cultural or religious differences among parents or health professionals. If used as reference for individual decisions, a treatment limit could have a needed focus on the importance to prioritize health care costs.

\section{BIG DATA IN NEONATOLOGY, TIME FOR A STANDARD}

T.G. Goos ${ }^{1,2}$, K.A.L. Mauff ${ }^{3}$, N. Bruining ${ }^{4}$, R.C.J. de Jonge ${ }^{1}$, D. Rizopoulos ${ }^{3}$, I.K.M. Reiss ${ }^{1}$

${ }^{1}$ Neonatology, Sophia Children's hospital-Erasmus Medical Center, Rotterdam, The Netherlands ${ }^{2}$ BioMedical Engineering, Delft University of Technology, Delft, The Netherlands

${ }^{3}$ Biostatistics, Erasmus Medical Center, Rotterdam, The Netherlands

${ }^{4}$ Clinical and Experimental Information Systems, Erasmus MC, Rotterdam, The Netherlands

Background: Big data is a hot topic and provides an unprecedented opportunity to improve care on the neonatal intensive care unit. Personalization and predictive models can be developed as long as enough of the right data is available. But data acquisition is usually not implemented in a way that all the raw measurement data is stored. Let alone that all clinical events are registered.

Methods: Collecting all patient monitoring data together with the clinical events helps us to learn from our patients. It enables the development of more personalized 
care, better visualization of data and the development of dynamic predictive models. Systems like HERO (Medical Predictive Science Corporation), expediting the diagnosis and treatment of septic patients with a day, show the potential of such models.

Models developed in one center need to be validated in other centers in order to be truly useful. This is only possible with (international) collaborations. Standardization of data storage and annotation are crucial.

Results: The Erasmus MC - Sophia Children's Hospital has started with logging all the real-time data from their neonatal patients. The intention is to set up a European network together with other NICU's to enable to store and share big data and develop and validate dynamic predictive models.

Conclusions: Big data and predictive models might well be the next game changer in neonatology after surfactant and the pulse oximeter. But the full potential is only unlocked when collaborations are formed such that the developed models are widely applicable.

\section{CORE NEONATAL DATA ITEMS TO FACILITATE LARGE, SIMPLE AND EFFICIENT CLINICAL TRIALS}

\section{Gale ${ }^{1}$}

\section{${ }^{1}$ Neonatal Medicine, Imperial College London, UK}

Background: Much neonatal care is inadequately evidence based because high quality research is lacking. It is neither financially or technically feasible to tackle existing neonatal uncertainties using traditional clinical trials. An alternative methodology involves incorporating clinical trials into existing electronic health record systems to facilitate large, simple, efficient neonatal trials. Concerns about data quality have limited the development such trials to date. We aimed to identify core baseline and outcome items for neonatal trials. These will facilitate simple clinical trials, improve data synthesis in meta-analyses and make research more relevant to stakeholders such as parents and patients.

Methods: We systematically reviewed 1) baseline data items recorded in neonatal clinical trials published in highly cited general medical journals; 2) outcomes reported in all neonatal clinical trials published in the preceding 5 years; 3 ) qualitative outcomes reported by parents, patients and other stakeholders in relation to neonatal care.

Results: 15 core baseline data items were were used in $>20 \%$ of high impact neonatal trials. Clinical trials report a wide range of different outcomes with little consistency between trials; outcomes reported as important by parents, patients and other stakeholders are divergent to outcomes reported in neonatal clinical trials.

Conclusions: High impact neonatal trials share common data elements, which can be used for neonatal clinical trials. Outcomes are reported inconsistently in neonatal clinical trials and do not reflect the views of parents, patients and other stakeholders in neonatal care. A core outcome set in neonatal care is being developed to address these issues.

\section{PREMATURITY IN CYPRUS, A RISING CONCERN. CAN WE REDUCE THE RATE?}

\author{
C. Karaoli ${ }^{1}$
}

${ }^{I}$ NICU Arch. Makarios III Hosp., Nicosia, Cyprus

Background: Despite improved health in developed countries in recent years prematurity remains an important public-health problem that worsened in the past decade. The range is $5-18 \%$ across 183 countries, mean rate $8.6 \%$ in developed nations. Neonatal morbidity remains a serious concern with enormous costs to the society.

Aim of the study was to identify prematurity rate in Cyprus and risk factors in order to suggest measures for improvement.

Methods: In a retrospective epidemiological study we analyzed data from the perinatal report 2008-2014 Health monitoring Unit, Ministry of Health, Republic of Cyprus and from the NICU, Arch. Makarios III Hospital, the unique referral Center of the island including live births beyond the 23 th gestational week.

Results: Population 858 thousand at the end of 2013 compared with 865 thousand in 2012 in the Government controlled area of the Republic of Cyprus. According to the demographic report of the statistical service the Republic of Cyprus has 9 - 10 thousand deliveries/year with $40 \%$ in public and $60 \%$ in private hospitals. The percentage of birth giving women coming from the EU and other countries has exceeded the corresponding percentage of Cypriots. A steep rise in preterm births was noted during the last years of the study reaching $13 \%$ in 2014. Live births $<32$ weeks are rather steady around $3.7 \%$, rates of moderate preterms 32 - 36 weeks increased from 11 to $13.6 \%$. Cyprus has the highest overall rate of prematurity and the highest rate of multiples in Europe. Preterm labor is $7.5 \%$ for singletons, $61 \%$ for twins and $100 \%$ for triplets; $22.8 / 1000$ women deliver twins, $4.6 / 1000$ deliver triplets. Fertility treatment happens in $6.2 \%$ of mothers ( $1-3 \%$ in other countries). On average, $2.4 \%$ of women giving birth in the public sector became pregnant after some form of assisted reproduction with $1.9 \%$ in-vitro fertilization. The rate of cesarean section (CS) has a steady rise reaching $57 \%$ in 2014, planned CS $27.5 \%$. Among preterm births the percentage of CS was higher than among vaginal deliveries. More than $50 \%$ of preterms $<37$ weeks are admitted to a neonatal intensive care unit (NICU). Increase of admissions to NICU was from 7.4 to $13.4 \%$ with an increasing rate of admitted twins and triplets. The rate of late preterms admissions is 
rather stable with a trend of reduction last year. Fetal mortality rate at or after 22 completed weeks of gestation is $3.5-4.5$ per 1000 live births. Early, late, and post neonatal mortality rate at or after 23 completed weeks is $0.5,0.3,0.7$, respectively. Infant mortality rate is 1.6 per 1000 live births.

Conclusions: Prematurity is a worldwide crisis and places a great role emotionally, physically $\&$ financially on families, medical systems \& regional or national economies. Although preterm birth etiology is multifactorial, we believe that we can reduce the rate of prematurity in Cyprus by the following measures: 1) Awareness campaign, especially for non-Cypriot nationals for significance of preconceptual and early antenatal care. 2) Legislation to control assisted reproduction, discussed in Cyprus parliament for the last 15 years, voted in 2016. 3) Limit of embryo transfer to two embryos per cycle, ideally single embryo transfer like in Scandinavia. 4) Discourage elective cesarean sections and labor induction unless there is a compelling medical reason in order to reduce the number of late preterms. 5) Eliminate early elective cesarean section prior to 39 weeks. 6) Compulsory continuous medical care and mothers education. 7) Educate women - especially migrants - and health care workers managing pregnancies. 8) Prolong interval between pregnancies to 12-18 months. 9) Aim for appropriate weight and no smoking. 10) Improve the measures for predicting and preventing preterm birth.

\section{THE "HEALTHY IMMIGRANT PARADOX": EXPERIENCE IN A GREEK PUBLIC MATERNITY HOSPITAL}

\author{
E. Sdona ${ }^{1}$, E. Ragkou ${ }^{1}$, D.D. Briana ${ }^{1}$, \\ D. Papamichail ${ }^{2}$, T. Panagiotopoulos ${ }^{2}$, \\ A. Malamitsi-Puchner $^{1}$
}

\section{${ }^{1}$ Department of Neonatology, National and Kapodistrian University of Athens, Athens, Greece ${ }^{2}$ Department of Child Health, National School of Public Health, Athens, Greece}

Background: Lately, Greece received huge migrant flows. It is known that socioeconomic disparities related to immigration adversely affect health. Nevertheless, current studies refer to the "healthy immigrant paradox", implying more favorable outcomes in immigrants than in native counterparts. This study aimed to investigate immigration impact on several perinatal parameters.

Methods: Birth records (01/01/2005 - 31/12/2014) from an Athenian public Maternity Hospital, concerning maternal (ethnicity, age, delivery mode) and neonatal (gender, birth weight, gestational age) variables were analyzed using EpiData software. Immigrants were classified by country of origin in high, medium and low Human Development Index clusters; Greeks served as controls. Comparison of results between Greeks and immigrants in maternal subgroups of $<$ and $>35$ years was conducted.

Results: Almost one third of 14923 deliveries applied to immigrants. $31.6 \%$ of Greeks and $17.2 \%$ of immigrants $(p<0.001, \mathrm{RR}=0.54,95 \% \mathrm{CI}=0.54-0.55)$ delivered at $>35$ years, $9.7 \%$ of Greek and $6.2 \%$ of immigrant newborns weighted $<2500 \mathrm{~g}(p<0.001, \mathrm{RR}=0.64,95 \%$ $\mathrm{CI}=0.62-0.66) .10 .4 \%$ of Greek and $7.6 \%$ of immigrant neonates were born $<37$ weeks $(p<0.001, \mathrm{RR}=0.73,95 \%$ $\mathrm{CI}=0.70-0.75) .50 .9 \%$ of Greek and $44.4 \%$ of immigrant infants were born by caesarean section $(p<0.001$, $\mathrm{RR}=0.87,95 \% \mathrm{CI}=0.87-0.88)$.

Conclusions: The "healthy immigrant paradox", applies to immigrants originating from countries with low prevalence of lifestyle-associated behaviors (obesity, sedentary life, smoking, unhealthy diet), factors affecting mother/child health. Due to cultural and economic reasons, immigrant women deliver at an earlier age $(<35$ years), vaginally, with decreased rates of low birth weight and prematurity, thus presenting more favorable birth outcomes.

\section{EVALUATION OF ROP SCREENING CRITERIA IN SWITZERLAND AND PRELIMINARY RESULTS ON POSSIBLE NEW SCREENING CRITERIA}

Roland Gerull ${ }^{1}$, Dirk Bassler ${ }^{2}$, Bernard Laubscher ${ }^{3}$, Riccardo Pfister ${ }^{4}$, Mathias Nelle ${ }^{1}$, Beatrice Müller ${ }^{5}$, Christina Gerth-Kahlert ${ }^{6}$, Mark Adams ${ }^{2}$

${ }^{1}$ Inselspital Bern, Div.of Neonatology, 3008 Bern
${ }^{2}$ University Hospital Zurich, Neonatology, 8091
Zürich
${ }^{3}$ Hôpital Neuchâtelois, Pediatrics, 2000 Neuchâtel
${ }^{4}$ University Hospital Geneva, Neonatology, 1211
Geneva
${ }^{5}$ Ostschweizer Kinderspital St. Gallen, Intesive
Care and Neonatology, 9006 St. Gallen
${ }^{6}$ University Hospital Zürich, Ophthalmology, 8091
Zürich

Background: Retinopathy of prematurity (ROP) is a severe complication of preterm birth. Criteria for ROPScreening differ between countries as well as between units in Switzerland and usually include patients of less than 31 - 32 weeks of gestational age (GA) and a birthweight (BW) of less than $1250-1500 \mathrm{~g}$. We aim for evaluation of ROP incidence in Switzerland and to assess if new screening criteria might better identify patients at risk.

Methods: Study is based on a retrospective analysis of all patients hospitalized at Inselspital Bern between 2006 and 2010 as well as the national registry of very preterm infants in Switzerland during 2006 to 2014 (SwissNeoNet). Multivariable logistic regression analysis of 10 fold 
imputed data was performed to analyze occurrence of ROP and risk factors to assess the feasibility of evidence based new ROP screening guidelines for Switzerland.

Results: In Berne, 553 patients were eligible and 386 patients had at least one fundoscopy for diagnosis of ROP. Of these 386 patients $30.3 \%$ had ROP at any stage and $4.9 \%$ had ROP III ${ }^{\circ}$. No patients above $810 \mathrm{~g} \mathrm{BW}$ or $261 / 7$ weeks GA had to be treated for ROP. Therapy was initiated at 60 th day of life in one child and $\geq 74$ th day of life in all other children. of the 5824 eligible SwissNeoNet patients, data on treatment for ROP was missing in 1021 patients. Numbers of patients treated for ROP for GA as of 27, 28, 29,30 and 31 weeks, respectively, were 7/482 (1.4\%), $4 / 584(0.7 \%), 1 / 753(0.1 \%), 2 / 921(0.2 \%)$ and $0 / 1254$ $(0.0 \%)$. Logistic regression analysis of 10 fold imputed datasets for children born $<31$ weeks GA from 2006-2012 revealed variables GA, oxygen supply in days, CPAP in days, multiple birth (yes/no) and surfactant treatment (yes/ no) as predictors for ROP treatment. Their application on data collected in 2013 and 2014 predicted a reduction in the number of screened patients to $301 / 958$ (31.4\%) to reach a sensitivity of $91 \%$ (one patient not detected). However, 922/958 patients $(96.0 \%)$ would have required screening to reach a sensitivity of $100 \%$.

Conclusions: Rates of ROP treatment in Switzerland are very low ( $1.7 \%$ for children born below 32 weeks GA). This fact and the missing data on ROP treatment impede a highly sensitive prediction model to reduce screening criteria. However, it may be possible to optimize existing screening criteria based on risk factor analysis. Current efforts aim to retrieve missing data and optimize prospective data collection.

\section{FOLLOW-UP OF CHILDREN WITH TREATED RETINOPATHY OF PREMATURITY (ROP)}

$\underline{\text { H. Varendi }}^{1,2}$, A. Kannukene' ${ }^{3}$, P. Jürì ${ }^{2}$

${ }^{1}$ Department of Pediatrics, Institute of Clinical

Medicine, University of Tartu, Estonia

${ }^{2}$ Children's Clinic, Tartu University Hospital, Estonia

${ }^{3}$ Medical Faculty, University of Tartu, Estonia

Background: Treatment of ROP has changed, with concerns that new, anti-VEGF treatment may have unwanted systemic effects. We assessed the outcome of treated ROP in changing treatment methods and time.

Methods: The single center study included preterm infants, 32 gestational weeks (GW) at birth (VPTI) born in 2002 - 2016, treated for ROP. Child ophthalmologist collected ROP screening data prospectively for all infants born 22 - $32 \mathrm{GW}$. The information about ophthalmological and developmental outcome was obtained from the patient electronic database. Of 650 VPTI who survived until complete vascularization of retina and screened for ROP, 62 newborns [at birth median 25 (range 22 - 32) GW; weight $780(475$ - 1395) g], were allocated to ROP treatment. In 2002 - 2009 laser photocoagulation $(n=31) ; 2009$ - 2010 combined [laser and bevacizumab (anti-VEGF) intravitreal injection] $(\mathrm{n}=8) ; 2010$ - 2016 bevacizumab as monotherapy $(\mathrm{n}=23)$ was applied.

Results: In the laser group, at median age of 6 years, 11 children (35\%) had vision impairment (blind eye or visus $<0.2$ in 17 eyes) that affected also psychomotor development; additional 5 childrenhadneurodevelopmental impairment or died in infancy. In the combined treatment group, 2 children ( 2 eyes) had impaired vision and 3 neurodevelopmental impairment. In the bevacizumab group, at median age of 1.5 years, no eyes with vision impairment but 5 children had moderate developmental problems (22\%). Overall, combined negative outcome of VPTI was significantly lower $(p=0.04)$ in bevacizumab group.

Conclusion: Treatment with bevacizumab seems not to have negative impact on the outcome of VPTI. Vision impairment reduced significantly during 2002 - 2016, accompanied by decrease of psychomotor delay, at least by age 1.5 years.

\section{BEHAVIORAL TESTING IN THE RABBIT: CONSIDERATIONS REGARDING DEVELOPMENTAL STUDIES WITH LONG-TERM ASSESSMENT}

M. Bruschettini ${ }^{1}$, D.L.A. van den Hove $^{2}$, G. Gümüş ${ }^{3}$, O. Romantsik ${ }^{1}$, A.A. Agyemang ${ }^{1}$, M. Gram ${ }^{1}$, S. Vallius ${ }^{1}$, H. Karlsson ${ }^{1}$, R. Sandgren ${ }^{4}$, D. Ley ${ }^{1}$

${ }^{1}$ Lund University, Dept Pediatrics, Institute for Clinical Sciences, Lund, Sweden

${ }^{2}$ Maastricht University, School for Mental Health and Neuroscience (MHENS), Dept Psychiatry and Neuropsychology, Maastricht, The Netherlands ${ }^{3}$ Fetal $i+D$ Fetal Medicine Research Center, BCNatal-Barcelona Center for Maternal-Fetal and Neonatal Medicine (Hospital Clinic and Hospital San Juan de Deu), Institut Clinic de Ginecologia, Obstetricia i Neonatalogia, Institut d'Investigacions Biomediques August Pi i Sunyer, University of Barcelona, and Centre for Biomedical Research on Rare Diseases (CIBER-ER), Barcelona, Spain ${ }^{4}$ Lund University, Medical Faculty, Lund, Sweden

Background: In perinatal medicine, behavioral testing allows to assess the effects of interventions during gestation and the neonatal period on neurodevelopment. 
Many animal studies on brain development and impairment have been performed in the newborn rabbit because of its multiple similarities with the human. However, little is known about behavioral testing in the rabbit.

The aim of our study was to characterize a valid animal model for neonatal studies with long term assessment, including cognition and anxiety- related behavior.

Methods: Twenty-one adult rabbits ( 13 female, 8 male) were exposed to adapted versions of the open field test, the dark-light box test. In addition, they were subjected to object recognition testing with variations in inter-trialinterval, olfactory recognition and object location testing. Outcome measures from each test were analyzed by sex, litter size and body weight.

Results: Rabbits spent more time in exploring the novel versus the familiar object in both the olfactory (discrimination index $[\mathrm{D} 2]=0.34)$ and the visual $(\mathrm{D} 2=0.43)$ object recognition tests $(p=0.005)$. Rabbits could still discriminate the novel from the old object using $5,30(\mathrm{D} 2=0.71 ; p<0.001)$ and $90(\mathrm{D} 2=0.70 ; \mathrm{p}<0.001)$ minutes' inter-trial-intervals, whereas no recognition was observed after 24 hours' (D2=0.23). In addition, litter size $>3$ during the neonatal period was associated with increased explorative behavior and memory $(p=0.005)$, whereas body weight and sex were not.

Conclusions: We report settings and outcome measures for multiple behavioral tests, providing reference values and considerations for future developmental studies. Discrimination and memory in the rabbit seem to depend on litter size.

\section{CARDIOVASCULAR RISK PROFILE IN FORMER VERY PRETERM INFANTS AT A PRESCHOOL AGE}

\author{
U. Kiechl-Kohlendorfer $^{1}$, A. Posod ${ }^{1}$, I Odri \\ Komazec $^{1,2}$, D. Baumgartner ${ }^{1,3}$ \\ ${ }^{1}$ Department of Pediatrics II (Neonatology) and \\ ${ }^{2}$ Department of Pediatrics III (Pediatric \\ Cardiology, Pulmonology, Allergology and Cystic \\ Fibrosis), Medical University of Innsbruck, Austria \\ ${ }^{3}$ Department of Pediatrics, Clinical Division for \\ Cardiology, Medical University of Graz, Austria
}

Background: Cardiovascular disease is the leading cause of death worldwide. Evidence points towards an unfavorable cardiovascular risk profile of former preterm infants in adolescence and adulthood. Aim of the study was to determine whether cardiovascular risk predictors are already present at a preschool age.

Methods: We investigated five- to seven-year-old children born with a gestational age $<32$ weeks $(n=76)$ and a control group of same-aged children born at term $(n=79)$. Basic data were collected by means of follow-up databases and standardized questionnaires. At study visit, anthropometric data, blood pressure readings, blood samples after an overnight fast, aortic intima-media thickness and aortic elastic properties were obtained.

Results: Compared with children born at term, the preterm group showed significant differences in blood pressure readings, fasting glucose levels, homeostasis model assessment index and cholesterol levels $(p<0.05$, each). The preterm group showed significantly reduced distensibility and increased stiffness of the descending abdominal aorta $(p<0.05)$.

Conclusions: Clinical follow-up of preterm infants including cardiovascular parameters might be useful for tailoring early prevention programs and counteracting cardiovascular risk in adulthood.

\section{IMPACT OF PRETERM BIRTH ON RESPIRATORY MUSCLE FUNCTION AND EXERCISE CAPACITY IN CHILDHOOD}

$\underline{\text { G. Dimitriou }}^{1}$, A. Vervenioti ${ }^{1}$, S. Fouzas ${ }^{1}$

${ }^{1}$ Neonatal Intensive care Unit, Department of Pediatrics, University Hospital of Patras, Greece

Background: Studies in newborn infants have shown that respiratory muscle strength is influenced by maturation at birth. However, the long term impact of prematurity on respiratory muscle function, particularly in relation to exercise tolerance, remains unknown.

The aim of our study was to investigate whether prematurity affects respiratory muscle function at the long term, leading to reduced exercise capacity in childhood.

Methods: Thirty four 6 to 7 years-old children (mean age $6.4 \pm 0.2$ years), born at a gestational age of $35.5 \pm 3.4$ weeks (range 29 - 40), underwent lung function measurements, including determination of respiratory muscle function (maximal inspiratory pressure [MIP], pressure-time index of the respiratory muscles [PTimus]) and cardiopulmonary exercise testing (CPET) (total work [Wmax], maximal oxygen consumption $\left.\left[\mathrm{VO}_{2} \max \right]\right)$.

Results: In comparison to children born at term $(N=18)$, preterm-born subjects $(n=16)$ had lower MIP $(65 \pm 8.4$ vs. $\left.75.1 \pm 8.5 \mathrm{cmH}_{2} \mathrm{O} ; P=0.002\right)$, higher PTimus $(0.11 \pm 0.02$ vs. $0.09 \pm 0.02 ; P=0.014)$ and lower Wmax $(115.9 \pm 8.4 v s$. $124.1 \pm 7.2$ watts; $P=0.004)$. The $\mathrm{VO}_{2}$ max was comparable between the two groups (preterm 32.8 \pm 4.9 vs. $36.6 \pm 8.8 \mathrm{ml} / \mathrm{kg} / \mathrm{min} ; P=0.124)$. Gestational age $(\mathrm{GA})$ at birth was significantly correlated to MIP $(r=0.626$; $P<0.001)$, PTimus $(r=-0.433 ; P=0.039)$ and Wmax $(r=0.500 ; P=0.003)$. PTimus was significantly correlated with Wmax $(r=-0.877 ; \quad P<0.001)$ and $\mathrm{VO}_{2} \max$ $(r=-0.711 ; P<0.001)$. No direct correlation between GA and $\mathrm{VO}_{2}$ max was found $(r=0.249 ; P=0.156)$.

Conclusions: Preterm-born children exhibit compromised respiratory muscle function and decreased 
exercise tolerance as compared to children born at term. These findings suggest that, under demanding cardiopulmonary conditions, preterm-born children are more prone to respiratory muscle fatigue.

\section{OUTCOME PREDICTION IN PRETERM INFANTS WITH INTRAVENTRICULAR HEMORRHAGE USING A NEWLY DEFINED MRI BASED SCORING SYSTEM}

K. Goeral ${ }^{1}$, G. Kasprian ${ }^{2}$,T. Waldhoer ${ }^{3}$, R. Fuiko ${ }^{1}$, A. Berger ${ }^{1}$, M. Olischar ${ }^{1}, \underline{\text { K. Klebermass-Schrehof }}{ }^{1}$

${ }^{1}$ Division of Neonatology, Intensive Care and Neuropediatrics, Department of Pediatrics and Adolescent Medicine, Medical University of Vienna, Vienna, Austria

${ }^{2}$ Division of Neuroradiology and Musculoskeletal Radiology, Department of Radiology, Medical University of Vienna, Vienna, Austria

${ }^{3}$ Center of Public Health, Department of Epidemiology, Medical University of Vienna, Vienna, Austria

Background: Intraventricular hemorrhage (IVH) remains a significant morbidity in preterm infants. There is a wellknown correlation between IVH grade and neurodevelopmental outcome. However, so far no standardized scoring system taking into account size, severity and topography of injury is available.

The aim of the study was to create a MRI based scoring system by assessing all functional areas affected by the bleeding and analyze its correlation to outcome.

Methods: 83 neonates born $2000-2015$ with IVH grade II - IV who had MRI at term-equivalent age and assessment of neurodevelopmental outcome were included.

MRI scans were analyzed by the computation of a grey matter score (gyrus pre- and postcentralis, hippocampus, basal ganglia), white matter score (pyramidal tract, corpus callosum, radiatio optica, crossroad) and a total MRI score which included additional abnormalities (periventricular leukomalacia, hydrocephalus, cerebellar defects). Outcome was evaluated at 1,2 and 3 years via Bayley Scales of Infant development. The predictive ability of MRI scores was evaluated using receiver-operating curves and area under the curve (AUC).

Results: The AUC to distinguish between favorable and unfavorable outcome was better for psychomotor $(\mathrm{PDI}=0.81)$ compared to mental outcome $(\mathrm{MDI}=0.76)$. We were able to create a model, which allows outcome prediction based on a combination of MRI score and gestational age.
Conclusion: There is currently not much evidence with regard to severity and topography of injury when trying to predict outcome in preterm infants with IVH. The proposed score might fill this gap and serve as a prognostic tool with regard to long-term neurological outcome.

\section{THE PREMATCH STUDY: AN EFFORT TO QUANTIFY THE IMPACT PRETERM BIRTH ON CARDIOVASCULAR AND RENAL HEALTH}

\author{
A. Raaijmakers ${ }^{1}$, J. Staessen ${ }^{2}, \underline{\text { K. Allegaert }}{ }^{1,2}$ \\ ${ }^{1}$ Department of Development and Regeneration \\ ${ }^{2}$ Research Unit Hypertension and Cardiovascular \\ Epidemiology, Department of Cardiovascular \\ Sciences, KU, Leuven \\ ${ }^{3}$ Department of Pediatric Surgery and Intensive \\ Care, and Division of Neonatology Erasmus \\ MC-Sophia Children's hospitals, Rotterdam, The \\ Netherlands
}

Background and objectives: The PREMATurity as predictor of Children's cardiovascular and renal Health (PREMATCH) is a case-control study in former ELBW children (2000 - 2005) and controls (term) at the median age of 12 years to compare the phenotype, including body composition, renal function and cardiovascular assessment.

Methods: Growth (height, weight, head circumference, Z-scores), body composition (Bodystat), renal function (renal length, cystatin $\mathrm{C}$ converted to eGFR), cardiovascular assessment and retinal vascular aspects were assessed in former ELBW children and controls.

Results: Former ELBW children still have difficulties to reach their target height. ELBW adolescents show lower neurocognitive performance, grip strength and higher fat body mass. Catch-up growth for weight in ELBW children in the first two years of life was associated with lower fat body mass. Renal length and glomerular filtration rate (cystatin C) were $0.28 \mathrm{~cm}(95 \% \mathrm{CI} 0.09-0.47)$ and 11.5 $\mathrm{mL} / \mathrm{min} / 1.73 \mathrm{~m}^{2}(6.4-16.6)$ lower in cases. The odds of having systolic (pre)hypertension in former ELBW cases was $6.43(2.52-16.4)$ and $10.9(2.46-48.4)$ with a low renin mechanism. Microvascular retinal arteriolar narrowing is observed in former ELBW young adolescents.

Conclusions: The phenotype (growth, body composition, renal function, retinal microvascularization) of former ELBWs differs significantly from controls in early adolescence. All these findings reflect mechanisms related to a higher risk factor for adverse health outcomes in adulthood. 


\section{TWIN-TWIN-TRANSFUSION SYNDROME (TTTS): LONG-TERM DEVELOPMENT AFTER INTRAUTERINE LASER COAGULATION}

$\underline{\text { P. Bartmann }}^{1}$, B. Busch ${ }^{1}$, K. Hecher ${ }^{2}$, D. Mueller ${ }^{2}$

${ }^{1}$ Dept. of Neonatology, Research Group Longitudinal Studies, Univ. Hospital Bonn, Germany

${ }^{2}$ Clinic for Obstetrics and Prenatal Medicine, University Hospital Hamburg, Germany

Background: Intrauterine laser coagulation of inter-twin anastomoses is a causal therapy for TTTS. Yet developmental data up to mid-childhood are rare.

Methods: $193 / 203$ surviving children, being treated by laser coagulation between 1995 and 1999, were evaluated by parent questionnaires and their school reports from 4 th and 6th grade. 179 questionnaires (93\%) and 152 school reports (79\%) were analyzed and whenever possible compared to data from the whole German population.
Comparisons between former donors and recipients are presented.

Results: Comparing results for donors (D) and recipients (R), we found non-significant trends for later school entry for $\mathrm{D}(18.8 \%$ vs. $16 \%)$, and $\mathrm{R}$ attending 1.6 times more often high-school education compared to $\mathrm{D}$ at $6^{\text {th }}$ grade. Different kinds of handicaps were more often observed in R (31.3\% vs. $23.8 \%)$ than in D. These were more frequently of physical origin, while D had physical and mental handicaps.

No differences between $\mathrm{D}$ and $\mathrm{R}$ could be detected for age at school entry, attending special-needs school at $4^{\text {th }}$ grade, average grades in various school subjects, repeating a school grade, need for special support and behavioral problems diagnosed by a physician.

Comparing these results with data from the whole German population, it can be shown that $\mathrm{D}$ as well as $\mathrm{R}$ show a higher percentage for late school entry, a higher rate of handicaps and according to the parents estimates more behavioral problems.

Conclusions: This study shows only small differences for donors and recipients in their outcome at school age, investigated latest at 6 th grade. 